\title{
Morphological and Chemical Variation of Wild Sweet Chestnut (Castanea sativa Mill.) Populations
}

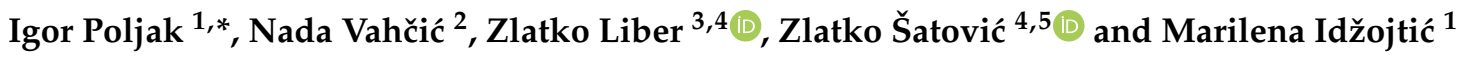 \\ 1 Institute of Forest Genetics, Dendrology and Botany, Faculty of Forestry and Wood Technology, \\ University of Zagreb, Svetošimunska cesta 23,HR-10000 Zagreb, Croatia; midzojtic@sumfak.hr \\ 2 Faculty of Food Technology and Biotechnology, University of Zagreb, Pierottijeva 6, \\ HR-10000 Zagreb, Croatia; nvahcic@pbf.hr \\ 3 Department of Biology, Faculty of Science, University of Zagreb, Marulićev trg 9a, HR-10000 Zagreb, Croatia \\ zlatko.liber@biol.pmf.hr \\ 4 Centre of Excellence for Biodiversity and Molecular Plant Breeding, Svetošimunska cesta 25, \\ HR-10000 Zagreb, Croatia; zsatovic@agr.hr \\ 5 Department for Seed Science and Technology, Faculty of Agriculture, University of Zagreb, \\ Svetošimunska cesta 25, HR-10000 Zagreb, Croatia \\ * Correspondence: ipoljak@sumfak.hr; Tel.: +385-1-2352547
}

Citation: Poljak, I.; Vahčić, N.; Liber, Z.; Šatović, Z.; Idžojtić, M. Morphological and Chemical Variation of Wild Sweet Chestnut (Castanea sativa Mill.) Populations. Forests 2022, 13, 55. https://doi.org/ 10.3390/f13010055

Academic Editor: Giovanbattista De Dato

Received: 21 November 2021 Accepted: 30 December 2021 Published: 3 January 2022

Publisher's Note: MDPI stays neutral with regard to jurisdictional claims in published maps and institutional affiliations.

Copyright: (C) 2022 by the authors. Licensee MDPI, Basel, Switzerland. This article is an open access article distributed under the terms and conditions of the Creative Commons Attribution (CC BY) license (https:// creativecommons.org/licenses/by/ $4.0 /)$.

\begin{abstract}
Sweet chestnut (Castanea sativa Mill., Fagaceae) is one of the oldest cultivated tree species in the Mediterranean, providing multiple benefits, and, since it has edible seeds, it represents an interesting model species for the research of morphological and chemical variability. In this study, morphometric methods and chemical analyses were used to quantify the extent of differences in phenotypic and nutritional traits between eight natural populations of sweet chestnut from different environmental conditions, where different management types are applied, high-forest and coppice. The samples were collected from the Prealps in Italy to the western part of Bosnia and Herzegovina. In total, 31 nut and kernel morphometric and nutritional traits were studied on 160 trees, and various multivariate statistical analyses were used to study intra- and interpopulation variations. Both analyses, morphometric and chemical, revealed a similar pattern of diversity, with morphological and chemical variability not associated with geographic or environmental variables. In addition, we found significant correlations between morphometric and chemical data. High phenotypic variability was determined both among and within the studied populations, and all populations had a similar level of diversity. The results of the analysis of morphological and chemical diversity can have many practical applications for the management, production, and conservation of the sweet chestnut genetic resources for nut production.
\end{abstract}

Keywords: fruits; population diversity; population structure; morphometric analysis; chemical analysis

\section{Introduction}

Sweet chestnut (Castanea sativa Mill.) is a medium-large, monoecious insect- and wind-pollinated deciduous tree that belongs to the family Fagaceae [1]. This species is characterized by oblong-lanceolate toothed leaves and spiky cupules containing one to three (seven) reddish-brown nuts with edible, creamy-white seeds, usually called kernels. It is distributed in the Mediterranean area, from the Caspian Sea to the Atlantic Ocean, from $51^{\circ}$ north latitude in southwestern Germany and southern England, to $37^{\circ}$ north longitude on Tlemcen Mountains in Algeria [2]. Due to its multiple benefits (wood, fruit, honey, and tannin), along with vine and olive, it is one of the oldest cultivated woody species; hence, it is hard to determine the exact boundaries of its natural distribution [3,4]. Nevertheless, pollen and plant macrofossil analyses suggest that the most probable natural range of sweet chestnut is delimited by several macro-regions [5]: the Transcaucasian region, northwestern Anatolia, the Apennines on the Italian Peninsula, the hills of the 
Prealps in northeastern Italy with a possible extension into Slovenia, the Cantabrian coast in the Iberian Peninsula, and the Balkan Peninsula, with one center in southern Greece and another one in Macedonia and southwestern Bulgaria. This was also confirmed by several genetic studies [6-12].

In its natural range, it grows at different altitudes, from $200 \mathrm{~m}$ all the way to $1800 \mathrm{~m}$, depending on the climate and latitude [13]. It does not grow in the lowlands as they are too humid and cold for it, nor in high mountainous regions, where there are great variations in daily and annual temperatures [14,15]. In general, sweet chestnut can be defined as a warmtemperate species that prefers acidic, deep, and well-drained soils [16]. It grows in areas where annual precipitation ranges from 750 to $1200 \mathrm{~mm}$, and mean annual temperature from $8^{\circ}$ to $15{ }^{\circ} \mathrm{C}$. Sweet chestnut avoids evergreen Mediterranean woods because they are too dry and too warm for it, as well as cold and foggy locations, and, in particular, cold valleys and frost pockets. In areas with warmer climate, it grows on northern and northeastern exposures and in humid valleys. Conversely, in northern regions and higher altitudes, it seeks sunny and warm habitats. Besides natural forests, we also find it in fruit orchards as grafted trees.

The origins of sweet chestnut growing and cultivation date back to ancient history, to the times of the ancient Greek and Roman civilizations [3]. Regardless of the reasons for its growing, for the wood or for nuts, intensive use of its forests is the main characteristic [15]. In the sweet chestnut distribution area, in addition to traditionally open stands composed of grafted chestnut trees (selected varieties) for fruit production, two main silviculture managing types may be identified [16,17]: high-forest and coppices. High-forest can be defined as chestnut stands that originate directly from seedlings without coppicing or grafting. In those stands, management follows longer rotation periods, 40-60 (100) years [18], and, because of that, the trees in those stands are of bigger dimensions. The wood obtained from high-forests is of high quality and can be used in the construction industry and manufacture of furniture. Still, regardless of its dimensions, its use can sometimes be limited due to the chestnut's susceptibility to the ring-shake. A more traditional type of management for sweet chestnut timber production is the coppice system. Coppice stands are usually pure chestnut forests where tree stems are repeatedly cut down and renewed from dormant buds of the stump. In this management type, a canopy is usually formed from multiple stems from a common stump. Due to high resprouting ability and remarkably fast growing, coppice represents the main type of forest management with about $80 \%$ of the chestnut forests for wood production [16,17]. The wood from such stands has a broad application in various activities, and is used as raw material for tannin and coal, as firewood, and in the manufacture of pillars, stakes, sticks, and various small items, such as barrels, shingles, sleepers, etc. Depending on the desired products, the silvicultural practices and rotation periods (5-30 years) for coppice stands are quite diverse. Regardless of the type of management mentioned above, whether high-forest or coppice, chestnut forests were used not only for timber production, but also for nut production.

Such a long-range distribution area of sweet chestnut throughout Europe and Asia Minor, with contrasting environmental conditions and varying silvicultural management practices, has significantly affected ecotypic differentiation, genetic polymorphism, and phenotypic plasticity. Several studies have been carried out so far, which established that environmental characteristics [10,19-21] and management type [22,23] can affect the genetic diversity of sweet chestnut populations. Martín et al. [20] highlighted the separation of sweet chestnut populations from the north and the south by EST-SSRs as the existence of differentiation in adaptive traits. Similar results were also reported by Míguez-Soto et al. [21] for Mediterranean and northern Iberian gene pools of wild sweet chestnut populations. The above-mentioned authors revealed that both gene pools identified in the Iberian Peninsula with neutral markers, Mediterranean and north, correspond to two different ecotypes, xeric and mesophytic, adapted to their climatic conditions as a consequence of natural selection caused by drought in the central and southern populations, and by cold temperatures in the north of the Iberian Peninsula. In addition, it was found that long-term management 
techniques may influence the genetic diversity of sweet chestnut populations [22]. However, when comparing managed coppice and naturalized stands, their results, in general, only showed slight (nonsignificant) differences in within-population genetic parameters, and weak, though significant, differences in the amount of two-locus disequilibrium [22]. Nevertheless, little is known about how management types and environmental conditions affect phenotypic and chemical properties of natural sweet chestnut populations. A large number of morphometric [24-31] and/or chemical [32-45] studies have been carried out to date on the nuts of grafted trees from orchards, while there are only a few studies that include a morphometric analysis of nuts from natural populations [46-51].

Given the context provided above, in the present study, we aim to contribute to the understanding of the morphological and chemical diversity of wild sweet chestnut populations. To do that, we collected samples from 160 trees originating from eight natural populations stretching from the Prealps in Italy to the western part of Bosnia and Herzegovina. In our study, we considered populations from different environmental conditions, continental and sub-Mediterranean biogeographical regions, where different management types are applied, high-forest and coppice. Our main objectives were to evaluate morphological diversity, kernel proximate composition, macro- and micronutrients, and population structure and to test the correlations between geographic, environmental, morphological, and chemical variation in wild sweet chestnut populations.

\section{Materials and Methods}

\subsection{Plant Material}

The material for morphometric and chemical analysis was collected from eight wild sweet chestnut populations (Table 1). The study encompassed five populations from Croatia and one population from Italy, Slovenia, and Bosnia and Herzegovina each. Basic information on the populations, such as sampling sites, biogeographical region, and management type, is shown in Table 1. Within each population, samples were collected from 20 trees, and approximately $1 \mathrm{~kg}$ of nuts was collected from each tree and subsequently used for morphometric and chemical analyses. Minimum distance between the trees from which the samples were collected was $50 \mathrm{~m}$, in order to avoid taking samples from related individuals.

Table 1. Acronyms, sample size (N), geographic co-ordinates, countries, biogeographical regions, and management types for eight sweet chestnut (Castanea sativa Mill.) populations.

\begin{tabular}{cccccccc}
\hline Acronym & Population & N & Latitude & Longitude & Country & $\begin{array}{c}\text { Biogeographical } \\
\text { Region }\end{array}$ & Management Type \\
\hline P01 & Combai & 20 & 12.056333 & 45.928222 & ITA & sub-Mediterranean & high-forest \\
P02 & Pazin & 20 & 13.916000 & 45.234361 & CRO & sub-Mediterranean & high-forest \\
P03 & Bosiljevo & 20 & 15.278500 & 45.415306 & CRO & continental & high-forest \\
P04 & Karlovac & 20 & 15.499583 & 45.481917 & CRO & continental & high-forest \\
P05 & Tisovec & 20 & 15.845389 & 46.270917 & SVN & continental & high-forest \\
P06 & Macelj & 20 & 15.836222 & 46.257139 & CRO & continental & high-forest \\
P07 & Petrova gora & 20 & 15.833917 & 45.318667 & CRO & continental & coppice \\
P08 & Cazin & 20 & 15.955278 & 44.980444 & BIH & continental & coppice \\
\hline
\end{tabular}

\subsection{Morphometric Analysis}

The morphometric analysis included only the nuts located laterally in cupules [46,48-51]. Ten nut traits (Figure S1) and eight ratios were examined on a total of 20 nuts from each tree to evaluate variation among and within sweet chestnut populations. First, nut mass was determined, and then the following characteristics were measured using a digital caliper: nut height $(\mathrm{NH})$, width $(\mathrm{NW})$, and thickness (NT); distance from nut base to the point of maximum nut width (PMNW); and hilum length (HL) and width (HW). Afterwards, the nuts were cut across at the widest point, the number of kernels (NK) and the number of intrusions of the seed coat into the kernel (NI) were counted, and the length of the longest intrusion of the seed coat into the kernel (LI) was measured. The following ratios were calculated from 
the measured characteristics: nut height/nut width $(\mathrm{NH} / \mathrm{NW})$, nut thickness/nut height $(\mathrm{NT} / \mathrm{NH})$, nut thickness/nut width $(\mathrm{NT} / \mathrm{NW})$, hilum length/nut width (HL/NW), hilum width/nut thickness (HW/NT), hilum width/hilum length (HW/HL), length of the longest intrusion of the seed coat into the kernel/nut thickness (LI/NT). In total, traits of 3200 nuts were measured and 57,600 simple data values were obtained and used in the statistical processing of data.

\subsection{Chemical Analysis}

Immediately after collection, the freshly picked nuts were peeled. The pericarp and seed coat were removed, leaving only the edible part of the nut remaining, that is the kernel. The kernels were then ground and homogenized using a hand-held blender, without losing moisture or volatile compounds [52]. A total of $50 \mathrm{~g}$ of ground sample from each tree was put into Ziploc PVC bags. The bags were labelled and placed into a freezer, at $-20{ }^{\circ} \mathrm{C}$ until further handling.

Water, ash, crude protein, and crude fat contents were determined according to the procedures established by the Association of Official Analytical Chemists (AOAC). All analyses were performed in duplicate. Water content was measured using the physical, indirect method of drying the sample at $105^{\circ} \mathrm{C}$ over $5 \mathrm{~h}$ until a constant mass was achieved [53]. Total mineral content was determined as ash content in the form of inorganic matter remaining after combustion of organic matter. A previously carbonized sample was combusted in a muffle oven using the dry combustion method at $580{ }^{\circ} \mathrm{C}$ until a uniform light grey ash was produced or the combusted matter showed constant mass [54]. To determine the crude fat content, previously weighed and dried chestnut samples were put into the Soxhlet apparatus and underwent continuous and repetitive extraction using organic solvents [55]. The extraction interval took $16 \mathrm{~h}$ and medicinal-grade petroleum was used as solvent. The Kjeldahl method was employed to determine the total nitrogen content [56], in combination with a copper catalyst using the block digestion system Foss Tecator 6-1007 Digestor and the Foss Kjeltec ${ }^{\mathrm{TM}} 8100$ Auto Distillation unit. Crude protein content was obtained by multiplying total nitrogen by a conversion factor of 5.30 . The carbohydrate content was estimated by subtracting all previously mentioned components from $100 \%$ [39, $40,44,45,57,58]$.

Macro- and micronutrients ( $\mathrm{K}, \mathrm{Ca}, \mathrm{Mg}, \mathrm{Na}, \mathrm{Cu}, \mathrm{Fe}, \mathrm{Mn}$, and $\mathrm{Zn}$ ) were determined by atomic absorption spectrometry using the Varian SpectrAA 220 spectrophotometer. Ash solution was prepared by dissolving cooled ash in nitric acid by heating the solution on the gas burner for approximately $30 \mathrm{~min}$. This enabled the minerals (metals) present in the ash to transform into nitrate form, which is preferred for further analysis. The ash solution was transferred into a volumetric flask of known volume and filled up to the mark with deionized water.

\subsection{Statistical Analysis}

Descriptive statistics, including arithmetic mean, standard deviation, and coefficient of variation, were calculated for the particular morphometric and chemical traits for each population in order to determine the range of their variation [59]. To determine intra- and interpopulation nut morphological variation, hierarchical analysis of the variance (ANOVA) was used. The analyzed factors of variability were population and tree by nesting the tree factor into the population factor. Univariate analysis of variance was performed to test for differences in the mean values between the studied populations for 13 chemical traits. Descriptive statistics and ANOVA analyses were carried out using the program package STATISTICA [60].

Several multivariate analyses were used to examine the morphological and chemical diversity of the studied populations: the analyses were performed separately for morphological data and for chemical data. Firstly, principal component analysis was used to calculate the principal components across all individuals and all studied morphometric and chemical traits. Biplots were constructed by two principal components showing the an- 
alyzed individuals and traits. In addition, discriminant analysis was performed to evaluate the utility and importance of the studied morphological and chemical traits by determining which traits were most useful for maximizing population differentiation. Classification discriminant analyses were then used to determine the proportion of individuals that were correctly classified into the studied populations. A cross-validation testing procedure was performed prior to the classification discriminant analysis. In cross-validation, five individuals were removed from the original dataset. Finally, a canonical discriminant analysis was performed based on the minimal dataset of traits that differentiated best between the studied populations. In addition, a cluster analysis was employed to obtain an overall view of the structure of the data using populations as operational taxonomic units (OTUs). Pairwise Euclidean distances were calculated, and cluster analysis was performed using the unweighted pair-group method with arithmetic mean (UPGMA). Finally, the results of cluster analysis were plotted in the form of dendrograms. The above multivariate statistical analyses were conducted using the statistical program R [61].

To test correlation between morphometric, chemical, geographic, and environmental data, four different matrices were calculated. Climate data from the WorldClim 2 database with a spatial resolution close to one square kilometer were used to calculate the environmental distance matrix $[62,63]$. Environmental differences were calculated as Euclidian distance between population means for the first three principal components of the PC analysis. Squared Mahalanobis distances between populations were computed to obtain a matrix of morphometric and chemical distances among populations. Geographic distances were calculated from the latitude and longitude of the site of sample collection. Finally, to assess isolation by distance (IBD) and isolation by environment (IBE), response matrices (morphological differences and chemical differences) were compared to the two predictor matrices (climate differences and geographic distance) using simple Mantel tests [64-66]. In addition, Mantel test was used to test the correlations between morphological and chemical distance matrices. The significance level was assessed after 10,000 permutations, as implemented in NTSYS-pc Ver. 2.02 [67].

\section{Results}

\subsection{Nut Morphometric Traits}

The results of the performed statistical analysis are shown in Table 2, by population $(\mathrm{N}=400)$ and overall for all populations together $(\mathrm{N}=3200)$. The average mass of the nuts for the eight studied populations was $7.87 \mathrm{~g}$. Variability coefficients for nut mass ranged from $24.59 \%$ for the Karlovac population to $35.84 \%$ for the Bosiljevo population. Among the studied populations, on average, populations Macelj (9.44 g) and Pazin (9.39 g) had the highest nut mass, followed by the populations Tisovec (8.82 g), Bosiljevo (8.33 g), Combai (8.17 g), and Karlovac (7.96 g), and populations of Petrova gora (5.94 g) and Cazin (4.96 g) with the lowest nut mass on average. The Petrova gora and Cazin populations were also characterized by the lowest average measured values for nut height (NH), nut width (NW), and nut thickness (NT), as well as for the distance from the base to the largest section of the nut (PMNW) and hilum length (HL). The highest average values for nut width (NW) and thickness (NT), and hilum length (HL) and width (HW) were exhibited by the Macelj population. The longest nuts on average, with the narrowest hilum, were from the Pazin population. In general, the coppice populations were characterized by smaller nuts. 
Table 2. Descriptive statistical parameters for studied morphometric traits. M-arithmetic mean; SDstandard deviation; CV—coefficient of variation (\%). Acronyms of populations: P01-Combai; P02Pazin; P03-Bosiljevo; P04-Karlovac; P05-Tisovec; P06-Macelj; P07-Petrova gora; P08-Cazin.

\begin{tabular}{|c|c|c|c|c|c|c|c|c|c|c|}
\hline Trait & $\begin{array}{l}\text { Descriptive } \\
\text { Parameters }\end{array}$ & P01 & P02 & P03 & P04 & P05 & P06 & P07 & P08 & Total \\
\hline \multirow{3}{*}{ nut mass } & $\mathrm{M}$ & 8.17 & 9.39 & 8.33 & 7.96 & 8.82 & 9.44 & 5.94 & 4.96 & 7.87 \\
\hline & SD & 2.60 & 3.09 & 2.98 & 1.96 & 2.64 & 2.84 & 2.11 & 1.68 & 2.94 \\
\hline & $\mathrm{CV}$ & 31.78 & 32.86 & 35.84 & 24.59 & 29.87 & 30.06 & 35.55 & 33.89 & 37.35 \\
\hline \multirow{3}{*}{ nut height (NH) } & $\mathrm{M}$ & 24.85 & 26.90 & 26.15 & 25.48 & 26.72 & 26.17 & 23.31 & 23.40 & 25.37 \\
\hline & SD & 2.49 & 2.58 & 2.93 & 2.03 & 2.90 & 2.67 & 2.72 & 3.05 & 2.99 \\
\hline & $\mathrm{CV}$ & 10.00 & 9.60 & 11.22 & 7.97 & 10.86 & 10.19 & 11.67 & 13.03 & 11.77 \\
\hline \multirow{3}{*}{ nut width (NW) } & $M$ & 27.97 & 29.95 & 28.92 & 28.64 & 28.56 & 30.07 & 25.09 & 22.96 & 27.77 \\
\hline & SD & 3.70 & 3.72 & 4.16 & 2.96 & 3.42 & 3.38 & 3.39 & 2.75 & 4.16 \\
\hline & $\mathrm{CV}$ & 13.22 & 12.43 & 14.40 & 10.34 & 11.96 & 11.25 & 13.52 & 11.96 & 14.98 \\
\hline \multirow{3}{*}{$\begin{array}{l}\text { distance from base to } \\
\text { the point of maximum } \\
\text { nut width (PMNW) }\end{array}$} & $\mathrm{M}$ & 11.31 & 11.85 & 12.40 & 12.17 & 11.55 & 12.17 & 10.50 & 10.79 & 11.59 \\
\hline & SD & 1.65 & 1.54 & 1.63 & 1.37 & 1.57 & 1.54 & 1.48 & 1.71 & 1.69 \\
\hline & $\mathrm{CV}$ & 14.56 & 13.00 & 13.16 & 11.22 & 13.61 & 12.67 & 14.09 & 15.82 & 14.56 \\
\hline \multirow{3}{*}{ nut thickness (NT) } & $M$ & 18.04 & 17.71 & 16.82 & 17.13 & 18.30 & 18.91 & 14.94 & 14.20 & 17.01 \\
\hline & SD & 2.62 & 2.90 & 2.72 & 2.36 & 2.83 & 2.79 & 2.60 & 2.17 & 3.05 \\
\hline & $\mathrm{CV}$ & 14.50 & 16.40 & 16.16 & 13.78 & 15.49 & 14.76 & 17.37 & 15.26 & 17.92 \\
\hline \multirow{3}{*}{ hilum length (HL) } & $M$ & 21.35 & 20.17 & 22.06 & 21.81 & 20.55 & 23.83 & 19.73 & 17.86 & 20.92 \\
\hline & SD & 4.50 & 3.42 & 3.82 & 3.34 & 3.56 & 3.26 & 3.24 & 3.01 & 3.91 \\
\hline & $\mathrm{CV}$ & 21.07 & 16.96 & 17.33 & 15.34 & 17.31 & 13.69 & 16.40 & 16.88 & 18.70 \\
\hline \multirow{3}{*}{ hilum width (HW) } & $\mathrm{M}$ & 10.86 & 9.08 & 10.25 & 10.50 & 9.90 & 11.75 & 9.93 & 9.15 & 10.18 \\
\hline & SD & 2.69 & 1.83 & 1.99 & 2.07 & 1.94 & 1.96 & 2.00 & 1.97 & 2.22 \\
\hline & $\mathrm{CV}$ & 24.78 & 20.15 & 19.37 & 19.75 & 19.60 & 16.66 & 20.10 & 21.53 & 21.85 \\
\hline \multirow{3}{*}{ number of kernels (NK) } & M & 1.01 & 1.01 & 1.01 & 1.09 & 1.03 & 1.01 & 1.01 & 1.01 & 1.02 \\
\hline & SD & 0.07 & 0.07 & 0.10 & 0.30 & 0.17 & 0.07 & 0.10 & 0.10 & 0.14 \\
\hline & $\mathrm{CV}$ & 7.04 & 7.04 & 9.88 & 27.37 & 16.60 & 7.04 & 9.88 & 9.88 & 14.16 \\
\hline \multirow{3}{*}{$\begin{array}{c}\text { number of intrusions of } \\
\text { the seed coat into the } \\
\text { kernel (NI) }\end{array}$} & $M$ & 3.26 & 3.67 & 3.26 & 2.61 & 4.59 & 3.36 & 3.14 & 2.54 & 3.30 \\
\hline & SD & 2.08 & 1.95 & 1.70 & 1.88 & 1.75 & 1.89 & 1.97 & 1.85 & 1.98 \\
\hline & $\mathrm{CV}$ & 63.71 & 53.26 & 52.03 & 71.99 & 38.27 & 56.45 & 62.80 & 72.65 & 59.85 \\
\hline \multirow{3}{*}{$\begin{array}{l}\text { length of the longest } \\
\text { intrusion of the seed } \\
\text { coat into the kernel (LI) }\end{array}$} & $M$ & 4.04 & 4.88 & 4.70 & 3.79 & 6.15 & 5.73 & 3.96 & 2.71 & 4.50 \\
\hline & SD & 2.52 & 2.59 & 2.28 & 2.31 & 2.19 & 2.68 & 2.19 & 1.84 & 2.55 \\
\hline & $\mathrm{CV}$ & 62.50 & 52.99 & 48.52 & 60.94 & 35.55 & 46.75 & 55.42 & 67.96 & 56.83 \\
\hline \multirow{3}{*}{$\mathrm{NH} / \mathrm{NW}$} & $\mathrm{M}$ & 0.90 & 0.91 & 0.91 & 0.89 & 0.94 & 0.87 & 0.93 & 1.02 & 0.92 \\
\hline & SD & 0.08 & 0.09 & 0.08 & 0.07 & 0.09 & 0.07 & 0.07 & 0.11 & 0.09 \\
\hline & $\mathrm{CV}$ & 8.96 & 9.56 & 9.00 & 7.83 & 9.30 & 8.21 & 7.25 & 10.28 & 10.03 \\
\hline \multirow{3}{*}{$\mathrm{PMNW} / \mathrm{NH}$} & M & 0.45 & 0.44 & 0.47 & 0.48 & 0.43 & 0.46 & 0.45 & 0.46 & 0.46 \\
\hline & SD & 0.05 & 0.04 & 0.04 & 0.04 & 0.04 & 0.04 & 0.04 & 0.04 & 0.04 \\
\hline & $\mathrm{CV}$ & 10.26 & 8.90 & 7.60 & 8.49 & 8.92 & 7.68 & 9.32 & 8.51 & 9.28 \\
\hline \multirow{3}{*}{$\mathrm{NT} / \mathrm{NH}$} & $\mathrm{M}$ & 0.73 & 0.66 & 0.64 & 0.67 & 0.69 & 0.72 & 0.64 & 0.61 & 0.67 \\
\hline & SD & 0.10 & 0.08 & 0.08 & 0.09 & 0.09 & 0.09 & 0.09 & 0.09 & 0.10 \\
\hline & $\mathrm{CV}$ & 13.37 & 12.61 & 12.72 & 13.19 & 13.00 & 12.56 & 13.33 & 14.59 & 14.33 \\
\hline \multirow{3}{*}{ NT/NW } & $\mathrm{M}$ & 0.65 & 0.59 & 0.58 & 0.60 & 0.64 & 0.63 & 0.60 & 0.62 & 0.61 \\
\hline & SD & 0.07 & 0.06 & 0.05 & 0.07 & 0.07 & 0.08 & 0.07 & 0.07 & 0.07 \\
\hline & $\mathrm{CV}$ & 11.48 & 10.41 & 9.42 & 11.08 & 11.16 & 11.99 & 11.22 & 11.63 & 11.72 \\
\hline \multirow{3}{*}{ HL/NW } & M & 0.76 & 0.67 & 0.76 & 0.76 & 0.72 & 0.79 & 0.79 & 0.78 & 0.75 \\
\hline & SD & 0.08 & 0.06 & 0.05 & 0.07 & 0.07 & 0.06 & 0.06 & 0.08 & 0.08 \\
\hline & $\mathrm{CV}$ & 10.10 & 8.49 & 6.15 & 9.66 & 10.04 & 7.94 & 7.54 & 10.50 & 10.18 \\
\hline \multirow{3}{*}{$\mathrm{HW} / \mathrm{NT}$} & $\mathrm{M}$ & 0.60 & 0.51 & 0.61 & 0.61 & 0.54 & 0.62 & 0.67 & 0.65 & 0.60 \\
\hline & SD & 0.10 & 0.06 & 0.06 & 0.08 & 0.07 & 0.08 & 0.10 & 0.11 & 0.10 \\
\hline & $\mathrm{CV}$ & 16.95 & 12.26 & 10.44 & 13.01 & 13.73 & 12.24 & 15.00 & 16.31 & 16.13 \\
\hline
\end{tabular}


Table 2. Cont.

\begin{tabular}{|c|c|c|c|c|c|c|c|c|c|c|}
\hline Trait & $\begin{array}{l}\text { Descriptive } \\
\text { Parameters }\end{array}$ & P01 & P02 & P03 & P04 & P05 & P06 & P07 & P08 & Total \\
\hline \multirow{3}{*}{$\mathrm{HW} / \mathrm{HL}$} & $\mathrm{M}$ & 0.51 & 0.45 & 0.47 & 0.48 & 0.48 & 0.49 & 0.50 & 0.51 & 0.49 \\
\hline & SD & 0.09 & 0.06 & 0.06 & 0.06 & 0.06 & 0.06 & 0.06 & 0.08 & 0.07 \\
\hline & $\mathrm{CV}$ & 16.70 & 12.46 & 13.34 & 12.41 & 11.51 & 12.00 & 11.36 & 15.81 & 14.05 \\
\hline \multirow{3}{*}{$\mathrm{LI} / \mathrm{NT}$} & $\mathrm{M}$ & 0.22 & 0.27 & 0.28 & 0.22 & 0.34 & 0.30 & 0.26 & 0.19 & 0.26 \\
\hline & SD & 0.13 & 0.13 & 0.12 & 0.13 & 0.11 & 0.13 & 0.13 & 0.12 & 0.13 \\
\hline & $\mathrm{CV}$ & 57.80 & 49.23 & 42.11 & 60.06 & 33.03 & 44.71 & 50.63 & 65.97 & 51.74 \\
\hline
\end{tabular}

On average, for all populations together, one nut contained one kernel each $(\mathrm{NK}=1.02)$. Variability coefficients for this trait ranged from $7.04 \%$ for the Combai, Pazin, and Macelj populations to $27.37 \%$ for the Karlovac population. The highest number of polyembryonic nuts was recorded in the Karlovac population. The average number of intrusions of the seed coat into the kernel on the largest section of the nut ranged from 2.61 in the Karlovac population to 4.59 in the Tisovec population. The average length of those intrusions for all populations was $19-34 \%$ of the average nut thickness (LI/NT $=0.19-0.34$ ).

The analysis of derived variables showed that the Cazin population has nuts approximately equally wide and high $(\mathrm{NH} / \mathrm{NW}=1.02)$, while the nuts of the other populations are, on average, wider than high. The derived ratios range from 0.87 for the Macelj population to 0.94 for the Tisovec population. From the ratio between the height from the base to the largest section of the nut and the nut height $(\mathrm{PMNW} / \mathrm{NH})$, we can conclude that the nuts in all studied populations are widest at $46 \%$ of their height. The ratio of nut thickness and height $(\mathrm{NT} / \mathrm{NH})$ is 0.67 on average for all populations, whereas the ratio of nut thickness and width (NT/NW) is 0.61 .

The variables of hilum length and width were compared with the nut width and thickness (HL/NW and HW/NT, respectively). For all the studied populations together, the HL/NW ratio is 0.75 and HW/NT ratio is 0.60 . The variables of hilum length and width were also put in a mutual relationship (HW/HL), which shows that the nuts of all populations have a hilum that is, on average, twice as long as wide $(\mathrm{HW} / \mathrm{HL}=0.49)$. In terms of the above traits, the Pazin population stands out, as it is characterized by the lowest values for the above variables.

The results of the hierarchical analysis of variance are shown in Table 3. The analysis showed that the populations and trees within the populations were statistically significantly different for all traits analyzed. For the majority of measured traits, it was demonstrated that the largest share of variability from the total variance is conditioned by the variability of trees within the population. Exceptions were nut thickness (NT) and distance from nut base to the point of maximum nut width (PMNW), where the variability among trees within populations was somewhat less than the variability of nuts within the tree, and variables NK, NI, and LI, where the component of residual variance accounts for the highest share of variability. The "effect of error" was greater than the "effect of tree" for the derived variables $\mathrm{PMNW} / \mathrm{NH}, \mathrm{NT} / \mathrm{NH}, \mathrm{NT} / \mathrm{NW}, \mathrm{HW} / \mathrm{HL}$, and $\mathrm{LI} / \mathrm{NT}$, and smaller for the variables $\mathrm{NH} / \mathrm{NW}, \mathrm{HL} / \mathrm{NW}$, and $\mathrm{HW} / \mathrm{NT}$.

Table 3. Results of the hierarchical analysis of variance for nut and kernel morphometric traits.

\begin{tabular}{cccccc}
\hline Trait & Variance Component & df & $\begin{array}{c}\text { \% } \\
\text { Variation }\end{array}$ & F & $p$ \\
\hline \multirow{3}{*}{ nut mass } & Among populations & 7 & 26.40 & 11.3574 & $<0.0001$ \\
& Within populations & 152 & 48.46 & 20.2724 & $<0.0001$ \\
& Error & & 25.14 & & \\
\hline
\end{tabular}


Table 3. Cont.

\begin{tabular}{|c|c|c|c|c|c|}
\hline Trait & Variance Component & df & $\begin{array}{c}\% \\
\text { Variation }\end{array}$ & $\mathbf{F}$ & $p$ \\
\hline \multirow{3}{*}{ nut height (NH) } & Among populations & 7 & 18.47 & 7.09504 & $<0.0001$ \\
\hline & Within populations & 152 & 58.30 & 26.0985 & $<0.0001$ \\
\hline & Error & & 23.23 & & \\
\hline \multirow{3}{*}{ nut width (NW) } & Among populations & 7 & 31.67 & 14.2809 & $<0.0001$ \\
\hline & Within populations & 152 & 45.40 & 20.7954 & $<0.0001$ \\
\hline & Error & & 22.93 & & \\
\hline \multirow{3}{*}{$\begin{array}{l}\text { distance from base to } \\
\text { the point of maximum } \\
\text { nut width (PMNW) }\end{array}$} & Among populations & 7 & 13.95 & 6.98548 & $<0.0001$ \\
\hline & Within populations & 152 & 42.23 & 10.6354 & $<0.0001$ \\
\hline & Error & & 43.82 & & \\
\hline \multirow{3}{*}{ nut thickness (NT) } & Among populations & 7 & 26.22 & 13.7966 & $<0.0001$ \\
\hline & Within populations & 152 & 36.45 & 11.2423 & $<0.0001$ \\
\hline & Error & & 37.33 & & \\
\hline \multirow{3}{*}{ hilum length (HL) } & Among populations & 7 & 17.28 & 6.89291 & $<0.0001$ \\
\hline & Within populations & 152 & 55.98 & 21.9393 & $<0.0001$ \\
\hline & Error & & 26.74 & & \\
\hline \multirow{3}{*}{ hilum width (HW) } & Among populations & 7 & 12.49 & 5.34088 & $<0.0001$ \\
\hline & Within populations & 152 & 54.21 & 17.2763 & $<0.0001$ \\
\hline & Error & & 33.30 & & \\
\hline \multirow{3}{*}{$\begin{array}{l}\text { number of kernels } \\
(\mathrm{NK})\end{array}$} & Among populations & 7 & 2.56 & 3.43539 & $<0.01$ \\
\hline & Within populations & 152 & 12.56 & 2.47944 & $<0.0001$ \\
\hline & Error & & 84.88 & & \\
\hline \multirow{3}{*}{$\begin{array}{l}\text { number of intrusions } \\
\text { of the seed coat into } \\
\text { the kernel (NI) }\end{array}$} & Among populations & 7 & 8.68 & 5.99596 & $<0.0001$ \\
\hline & Within populations & 152 & 28.45 & 5.52612 & $<0.0001$ \\
\hline & Error & & 62.87 & & \\
\hline \multirow{3}{*}{$\begin{array}{l}\text { length of the longest } \\
\text { intrusion of the seed } \\
\text { coat into the kernel (LI) }\end{array}$} & Among populations & 7 & 16.80 & 11.0424 & $<0.0001$ \\
\hline & Within populations & 152 & 27.92 & 6.05035 & $<0.0001$ \\
\hline & Error & & 55.28 & & \\
\hline \multirow{3}{*}{$\mathrm{NH} / \mathrm{NW}$} & Among populations & 7 & 21.28 & 9.30401 & $<0.0001$ \\
\hline & Within populations & 152 & 48.20 & 16.79345 & $<0.0001$ \\
\hline & Error & & 30.52 & & \\
\hline \multirow{3}{*}{$\mathrm{PMNW} / \mathrm{NH}$} & Among populations & 7 & 12.15 & 10.8916 & $<0.0001$ \\
\hline & Within populations & 152 & 17.54 & 3.49451 & $<0.0001$ \\
\hline & Error & & 70.31 & & \\
\hline \multirow{3}{*}{$\mathrm{NT} / \mathrm{NH}$} & Among populations & 7 & 15.79 & 9.23331 & $<0.0001$ \\
\hline & Within populations & 152 & 33.25 & 7.52340 & $<0.0001$ \\
\hline & Error & & 50.97 & & \\
\hline \multirow{3}{*}{$\mathrm{NT} / \mathrm{NW}$} & Among populations & 7 & 10.09 & 7.36716 & $<0.0001$ \\
\hline & Within populations & 152 & 25.21 & 4.89694 & $<0.0001$ \\
\hline & Error & & 64.70 & & \\
\hline \multirow{3}{*}{$\mathrm{HL} / \mathrm{NW}$} & Among populations & 7 & 23.67 & 10.2695 & $<0.0001$ \\
\hline & Within populations & 152 & 48.26 & 18.1867 & $<0.0001$ \\
\hline & Error & & 28.08 & & \\
\hline \multirow{3}{*}{$\mathrm{HW} / \mathrm{NT}$} & Among populations & 7 & 24.91 & 12.9752 & $<0.0001$ \\
\hline & Within populations & 152 & 37.88 & 11.1793 & $<0.0001$ \\
\hline & Error & & 37.21 & & \\
\hline \multirow{3}{*}{$\mathrm{HW} / \mathrm{HL}$} & Among populations & 7 & 8.44 & 5.97359 & $<0.0001$ \\
\hline & Within populations & 152 & 27.55 & 5.30367 & $<0.0001$ \\
\hline & Error & & 64.01 & & \\
\hline \multirow{3}{*}{$\mathrm{LI} / \mathrm{NT}$} & Among populations & 7 & 10.89 & 7.35714 & $<0.0001$ \\
\hline & Within populations & 152 & 28.17 & 5.62329 & $<0.0001$ \\
\hline & Error & & 60.94 & & \\
\hline
\end{tabular}




\subsection{Kernel Chemical Composition}

The proximate composition and macro- and micronutrients of wild sweet chestnut kernels are presented in Table 4. Total carbohydrates were the major compound in the sweet chestnut kernels, ranging from $88.70 \mathrm{~g}$ per $100 \mathrm{~g} \mathrm{dm}$ (Macelj population) to $90.77 \mathrm{~g}$ per $100 \mathrm{~g} \mathrm{dm}$ (Bosiljevo population). The values were relatively uniform, and the average coefficient of variation was very low $(\mathrm{CV}=1.21 \%)$. The mean water content value was $57.57 \%$, with a coefficient of variation of $4.51 \%$. In general, high-forest populations had somewhat lower water content values than the managed coppice populations. Crude protein and crude fat presented significantly lower values: average crude protein content was $4.26 \mathrm{~g}$ per $100 \mathrm{~g} \mathrm{dm}$, and average crude fat content was $2.86 \mathrm{~g}$ per $100 \mathrm{~g} \mathrm{dm}$. Both of these parameters were very variable. The coefficient of variation was $16.48 \%$ for crude protein content and $24.86 \%$ for crude fat content. Ash content values ranged from $2.67 \mathrm{~g}$ per $100 \mathrm{~g} \mathrm{dm}$ to $3.50 \mathrm{~g}$ per $100 \mathrm{~g} \mathrm{dm}$, with a mean value of $3.06 \mathrm{~g}$ per $100 \mathrm{~g} \mathrm{dm}$. Differences between the studied populations were confirmed for all studied proximate constitutes.

Table 4. Descriptive statistical parameters and level of significance for studied chemical traits. $\mathrm{M}$-arithmetic mean; SD—standard deviation; CV—coefficient of variation (\%), dm-dry matter. Acronyms of populations: P01-Combai; P02—Pazin; P03-Bosiljevo; P04-Karlovac; P05-Tisovec; P06-Macelj; P07-Petrova gora; P08-Cazin.

\begin{tabular}{|c|c|c|c|c|c|c|c|c|c|c|c|}
\hline Trait & $\begin{array}{l}\text { Descriptive } \\
\text { Parameters }\end{array}$ & P01 & P02 & P03 & P04 & P05 & P06 & P07 & P08 & Total & $p$ \\
\hline \multirow{3}{*}{$w$ (water) $/(\mathrm{g} / 100 \mathrm{~g})$} & $\mathrm{M}$ & 54.79 & 56.35 & 56.76 & 58.49 & 55.26 & 58.89 & 59.75 & 60.28 & 57.57 & \multirow{3}{*}{$<0.0001$} \\
\hline & SD & 1.60 & 1.61 & 1.91 & 2.05 & 2.48 & 1.64 & 1.17 & 1.29 & 2.60 & \\
\hline & $\mathrm{CV}$ & 2.91 & 2.85 & 3.36 & 3.51 & 4.49 & 2.78 & 1.96 & 2.15 & 4.51 & \\
\hline \multirow{3}{*}{$w($ ash $) /(\mathrm{g} / 100 \mathrm{~g} \mathrm{dm})$} & $\mathrm{M}$ & 2.76 & 2.94 & 2.90 & 3.03 & 2.67 & 3.37 & 3.29 & 3.50 & 3.06 & \multirow{3}{*}{$<0.0001$} \\
\hline & SD & 0.26 & 0.23 & 0.35 & 0.23 & 0.83 & 0.23 & 0.32 & 0.24 & 0.47 & \\
\hline & $\mathrm{CV}$ & 9.28 & 7.84 & 11.89 & 7.44 & 31.14 & 6.70 & 9.87 & 6.82 & 15.39 & \\
\hline \multirow{3}{*}{$w$ (crude fat) $/(\mathrm{g} / 100 \mathrm{~g} \mathrm{dm})$} & $\mathrm{M}$ & 2.85 & 3.45 & 2.34 & 3.23 & 3.14 & 3.19 & 2.05 & 2.67 & 2.86 & \multirow{3}{*}{$<0.0001$} \\
\hline & SD & 0.56 & 0.65 & 0.39 & 0.58 & 0.82 & 0.45 & 0.47 & 0.47 & 0.71 & \\
\hline & $\mathrm{CV}$ & 19.60 & 18.91 & 16.55 & 18.10 & 26.05 & 13.97 & 22.72 & 17.44 & 24.86 & \\
\hline \multirow{3}{*}{$w$ (crude protein $) /(\mathrm{g} / 100 \mathrm{~g} \mathrm{dm})$} & M & 4.39 & 4.24 & 3.99 & 4.48 & 3.73 & 4.73 & 3.89 & 4.65 & 4.26 & \multirow{3}{*}{$<0.0001$} \\
\hline & SD & 0.61 & 0.28 & 0.50 & 0.87 & 0.46 & 0.71 & 0.85 & 0.51 & 0.70 & \\
\hline & $\mathrm{CV}$ & 13.80 & 6.51 & 12.45 & 19.47 & 12.24 & 14.92 & 21.88 & 11.08 & 16.48 & \\
\hline \multirow{3}{*}{$\begin{array}{c}w(\text { total carbohydrates }) / \\
(\mathrm{g} / 100 \mathrm{~g} \mathrm{dm})\end{array}$} & $\mathrm{M}$ & 90.00 & 89.37 & 90.77 & 89.26 & 90.47 & 88.70 & 90.76 & 89.18 & 89.82 & \multirow{3}{*}{$<0.0001$} \\
\hline & SD & 0.87 & 0.71 & 0.63 & 0.97 & 0.99 & 0.68 & 0.85 & 0.72 & 1.09 & \\
\hline & $\mathrm{CV}$ & 0.96 & 0.80 & 0.69 & 1.09 & 1.09 & 0.77 & 0.93 & 0.81 & 1.21 & \\
\hline \multirow{3}{*}{$w(\mathrm{Cu}) /(\mathrm{mg} / 100 \mathrm{~g} \mathrm{dm})$} & $\mathrm{M}$ & 0.85 & 0.94 & 0.91 & 0.84 & 0.79 & 0.86 & 0.87 & 0.75 & 0.85 & \multirow{3}{*}{$<0.0001$} \\
\hline & SD & 0.09 & 0.12 & 0.11 & 0.06 & 0.03 & 0.08 & 0.11 & 0.15 & 0.11 & \\
\hline & $\mathrm{CV}$ & 10.59 & 12.74 & 12.42 & 7.61 & 4.31 & 9.39 & 12.98 & 20.38 & 13.46 & \\
\hline \multirow{3}{*}{$w(\mathrm{Zn}) /(\mathrm{mg} / 100 \mathrm{~g} \mathrm{dm})$} & M & 1.28 & 1.39 & 1.35 & 1.35 & 1.34 & 1.38 & 1.24 & 1.13 & 1.31 & \multirow{3}{*}{$<0.0001$} \\
\hline & SD & 0.12 & 0.21 & 0.17 & 0.08 & 0.15 & 0.19 & 0.24 & 0.18 & 0.19 & \\
\hline & $\mathrm{CV}$ & 9.17 & 15.25 & 12.65 & 6.04 & 10.98 & 14.05 & 19.15 & 16.11 & 14.49 & \\
\hline \multirow{3}{*}{$w(\mathrm{Fe}) /(\mathrm{mg} / 100 \mathrm{~g} \mathrm{dm})$} & M & 1.69 & 1.70 & 1.79 & 1.19 & 1.50 & 1.51 & 1.48 & 1.65 & 1.56 & \multirow{3}{*}{$<0.0001$} \\
\hline & SD & 0.10 & 0.28 & 0.34 & 0.23 & 0.19 & 0.26 & 0.30 & 0.51 & 0.34 & \\
\hline & $\mathrm{CV}$ & 5.80 & 16.35 & 19.24 & 19.35 & 12.43 & 17.15 & 20.48 & 30.75 & 21.74 & \\
\hline \multirow{3}{*}{$w(\mathrm{Mn}) /(\mathrm{mg} / 100 \mathrm{~g} \mathrm{dm})$} & $\mathrm{M}$ & 8.89 & 9.80 & 11.52 & 12.54 & 3.58 & 3.42 & 9.79 & 14.15 & 9.21 & \multirow{3}{*}{$<0.0001$} \\
\hline & SD & 2.98 & 1.97 & 1.96 & 3.81 & 1.66 & 1.12 & 2.45 & 3.15 & 4.42 & \\
\hline & $\mathrm{CV}$ & 33.51 & 20.10 & 17.01 & 30.37 & 46.47 & 32.85 & 25.02 & 22.27 & 47.99 & \\
\hline \multirow{3}{*}{$w(\mathrm{Na}) /(\mathrm{mg} / 100 \mathrm{~g} \mathrm{dm})$} & $\mathrm{M}$ & 9.29 & 11.37 & 10.68 & 9.16 & 12.65 & 7.39 & 8.67 & 11.66 & 10.11 & \multirow{3}{*}{$<0.0001$} \\
\hline & SD & 3.00 & 4.67 & 4.17 & 2.37 & 2.52 & 1.63 & 2.04 & 2.24 & 3.37 & \\
\hline & $\mathrm{CV}$ & 32.29 & 41.08 & 39.06 & 25.89 & 19.91 & 22.10 & 23.50 & 19.21 & 33.33 & \\
\hline \multirow{3}{*}{$w(\mathrm{Ca}) /(\mathrm{mg} / 100 \mathrm{~g})$} & M & 100.03 & 177.03 & 183.02 & 108.51 & 90.22 & 118.67 & 147.88 & 160.74 & 135.76 & \multirow{3}{*}{$<0.0001$} \\
\hline & SD & 31.28 & 41.56 & 45.63 & 27.40 & 8.45 & 39.64 & 24.75 & 32.81 & 46.94 & \\
\hline & $\mathrm{CV}$ & 31.27 & 23.47 & 24.93 & 25.25 & 9.37 & 33.40 & 16.74 & 20.41 & 34.58 & \\
\hline
\end{tabular}


Table 4. Cont.

\begin{tabular}{|c|c|c|c|c|c|c|c|c|c|c|c|}
\hline Trait & $\begin{array}{l}\text { Descriptive } \\
\text { Parameters }\end{array}$ & P01 & P02 & P03 & P04 & P05 & P06 & P07 & P08 & Total & $p$ \\
\hline \multirow{3}{*}{$w(\mathrm{Mg}) /(\mathrm{mg} / 100 \mathrm{~g} \mathrm{dm})$} & M & 123.35 & 145.87 & 144.93 & 156.03 & 134.09 & 179.40 & 193.69 & 198.41 & 159.47 & \multirow{3}{*}{$<0.0001$} \\
\hline & SD & 15.50 & 19.76 & 27.12 & 22.60 & 10.69 & 36.52 & 27.72 & 23.86 & 35.24 & \\
\hline & $\mathrm{CV}$ & 12.57 & 13.54 & 18.71 & 14.48 & 7.97 & 20.36 & 14.31 & 12.03 & 22.10 & \\
\hline \multirow{3}{*}{$w(\mathrm{~K}) /(\mathrm{mg} / 100 \mathrm{~g} \mathrm{dm})$} & M & 2026.29 & 2209.35 & 1907.87 & 2134.40 & 1494.79 & 2514.16 & 2253.74 & 2468.82 & 2126.18 & \multirow{3}{*}{$<0.0001$} \\
\hline & SD & 269.01 & 336.41 & 300.68 & 314.04 & 174.84 & 582.53 & 264.67 & 173.35 & 441.53 & \\
\hline & $\mathrm{CV}$ & 13.28 & 15.23 & 15.76 & 14.71 & 11.70 & 23.17 & 11.74 & 7.02 & 20.77 & \\
\hline
\end{tabular}

All of the macro- and micronutrients varied significantly among studied populations. For macronutrients, K was the most abundant, ranging from $1907.87 \mathrm{mg}$ per $100 \mathrm{~g} \mathrm{dm}$ in the Bosiljevo population to $2514.16 \mathrm{mg}$ per $100 \mathrm{~g} \mathrm{dm}$ in the Macelj population, with $2126.18 \mathrm{mg}$ per $100 \mathrm{~g} \mathrm{dm}$ as the mean value. The second most abundant macronutrient was $\mathrm{Mg}$, varying from $123.35 \mathrm{mg}$ per $100 \mathrm{~g} \mathrm{dm}$ in the Italian population Combai to $198.41 \mathrm{mg}$ per $100 \mathrm{~g} \mathrm{dm}$ in the Bosnia and Herzegovina population Cazin, with $159.47 \mathrm{mg}$ per $100 \mathrm{~g}$ $\mathrm{dm}$ as the mean value. The $\mathrm{Mg}$ content was followed by Ca content. In general, higher $\mathrm{Mg}$ content was characteristic of extensively managed coppice populations (P07 and P08) and the highest $\mathrm{Ca}$ content of high-forest populations (P02 and P03) characterized by the carbonate substrate, covered with a deep soil layer. Regarding the micronutrients, $\mathrm{Na}$ and Mn were the most abundant. Mean Na value was $10.11 \mathrm{mg}$ per $100 \mathrm{~g} \mathrm{dm}$, and Mn $9.21 \mathrm{mg}$ per $100 \mathrm{~g} \mathrm{dm}$. Other studied micronutrients had significantly lower average values. Mean Fe value was $1.56 \mathrm{mg}$ per $100 \mathrm{~g} \mathrm{dm}, \mathrm{Zn} 1.31 \mathrm{mg}$ per $100 \mathrm{~g} \mathrm{dm}$, and $\mathrm{Cu} 0.85 \mathrm{mg}$ per $100 \mathrm{~g}$ $\mathrm{dm}$. In general, the populations characterized by the carbonate substrate, covered with a deep soil layer showed higher levels of $\mathrm{Fe}$ and $\mathrm{Cu}$, and the coppice populations lower levels of Zn.

\subsection{Population Structure and Correlations between Morphological, Chemical, Geographical, and Environmental Distances}

Principal component (PC) analysis based on ten morphological traits revealed that the first two principal components explained $75.1 \%$ of the total variability (Figure 1, Table S1). All of the studied morphometric traits were in negative correlation with the first principal component, and the highest correlations were related to the nut and hilum dimensions. The number of intrusions into the kernels and their length were in positive correlation with the second principal component. The biplot constructed by the first two principal components is presented in Figure 1. Although the studied trees on the graph form a continuous cloud of data along the first PC axis, there is a tendency to separate the individuals according to their management type.

The first two components from the PC analysis of the chemical traits explained $28.6 \%$ and $16.1 \%$ of the total variation, respectively (Figure 2, Table S2). PC1 was negatively related to $\mathrm{K}$, water, ash, $\mathrm{Mg}, \mathrm{Ca}$, and crude protein content, and positively to total carbohydrate content. PC2 was positively correlated with $\mathrm{Ca}, \mathrm{Cu}, \mathrm{Na}, \mathrm{Fe}$ and total carbohydrate content, and negatively with crude protein content. The third, fourth, and fifth PC axes contributed substantially less to the overall variability, with $12.7 \%, 10.3 \%$, and $8.1 \%$, respectively. However, they all have Eigen values greater than 1 . Similar to the morphometric analysis, a trend of separation between the samples from coppice populations and the samples from high-forest populations could be observed along the first PC axis. 


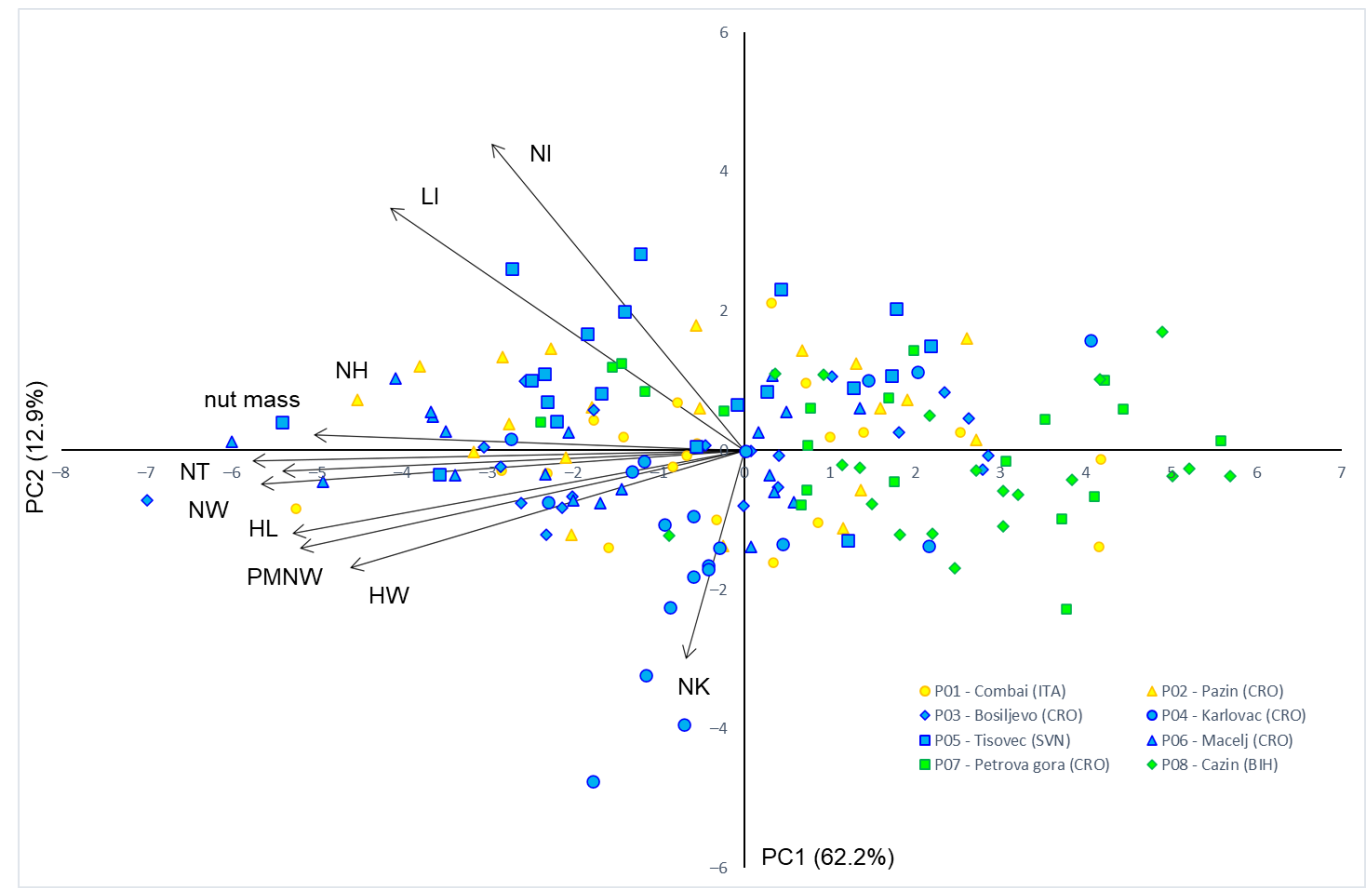

Figure 1. Principal component (PC) analysis of eight sweet chestnut populations based on ten morphological traits. The color of the signs is related to the biogeographical region and management type: yellow color-sub-Mediterranean high-forest populations; blue color-continental high-forest populations; and green color-continental coppice populations. Morphometric traits: nut height (NH); nut width (NW); distance from nut base to the point of maximum nut width (PMNW); nut thickness (NT); hilum length (HL); hilum width (HW); number of kernels (NK); number of intrusions of the seed coat into the kernel (NI); length of the longest intrusion of the seed coat into the kernel (LI).

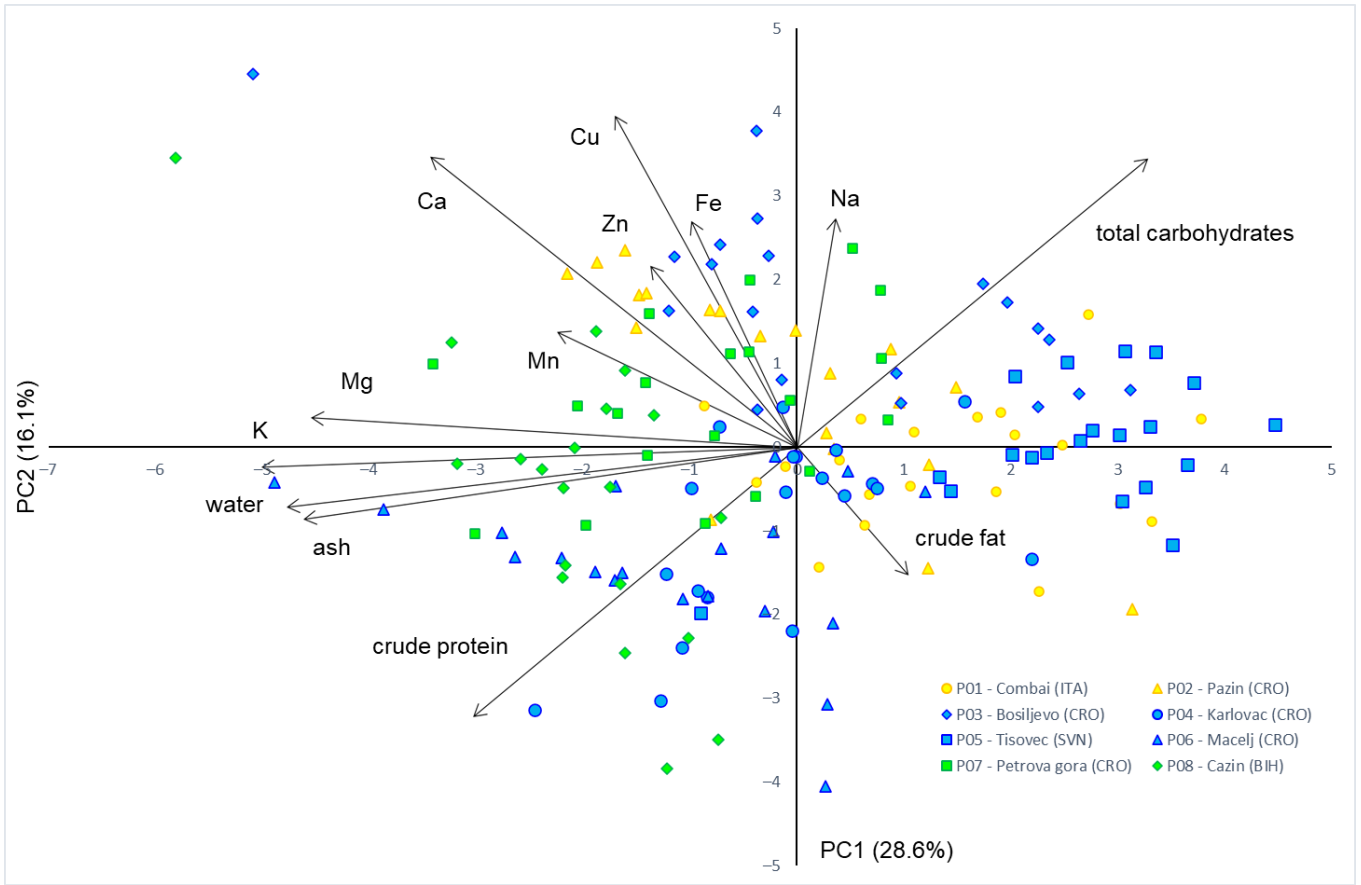

Figure 2. Principal component (PC) analysis of eight sweet chestnut populations based on 13 chemical traits. The color of the signs is related to the biogeographical region and management type: yellow color-sub-Mediterranean high-forest populations; blue color-continental high-forest populations; and green color-continental coppice populations. 
Eight out of ten morphological traits (distance from nut base to the point of maximum nut width (PMNW), nut width (NW), nut thickness (NT), kernels per nut (NK), length of the longest intrusion of the seed coat into the kernel (LI), nut mass, number of intrusions of the seed coat into the kernel (NI), and hilum width (HW)) were selected as the best discriminating factors between the studied sweet chestnut populations by stepwise discriminant analysis (Table S3). The discriminant function based on eight morphometric traits showed a classification success of $64.2 \%$. The classification of samples from the Petrova gora population was characterized by the lowest levels of accuracy (46.7\%), and the Bosnian and Herzegovinian population Cazin by the highest $(86.7 \%)$. The analysis based on eight traits revealed that the first canonical discriminant variate (CV1) explained $46.2 \%$ of the variation between populations. Although considerable overlap between studied populations in morphospace was revealed, samples from high-forest populations were grouped on the left side of the graph, and samples from coppice populations on the right side of the graph (Figure 3).

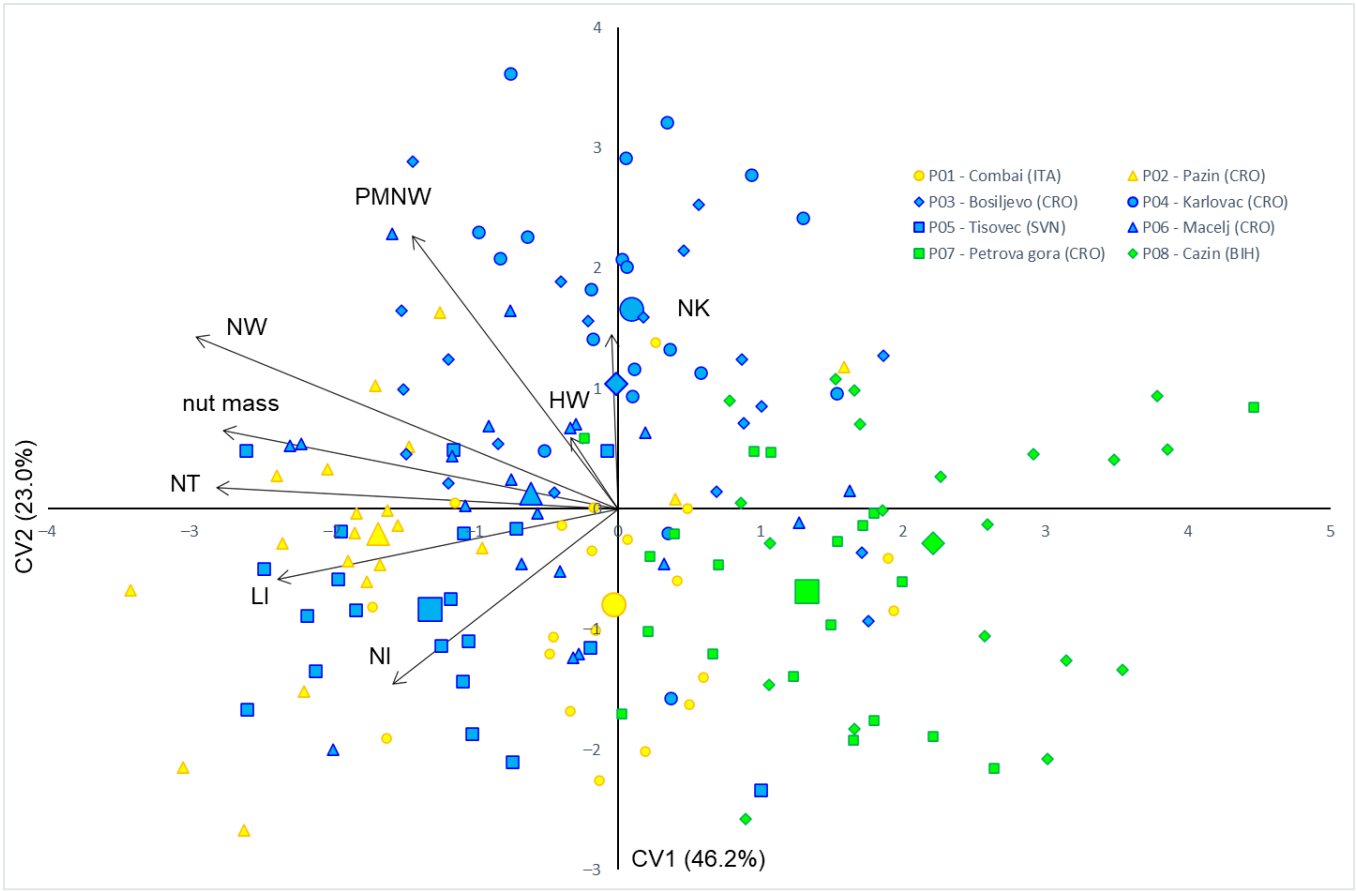

Figure 3. Discriminant analysis of eight sweet chestnut populations based on eight morphological traits that were the most useful for maximum discrimination between studied populations. Each individual tree is indicated by a small sign, while the population barycenters are represented by larger ones. The color of the signs is related to the biogeographical region and management type: yellow color-sub-Mediterranean high-forest populations; blue color-continental high-forest populations; and green color-continental coppice populations. Morphometric traits: nut width (NW); distance from nut base to the point of maximum nut width (PMNW); nut thickness (NT); hilum width (HW); number of kernels (NK); number of intrusions of the seed coat into the kernel (NI); length of the longest intrusion of the seed coat into the kernel (LI). Canonical discriminant variate (CV).

Regarding the kernel nutritional value, $\mathrm{Mn}, \mathrm{Mg}, \mathrm{Ca}, \mathrm{K}, \mathrm{Cu}$, crude fat, and water had the highest discrimination power between populations by stepwise discriminant analysis (Table S4). In terms of significance, the variables that follow are $\mathrm{Na}, \mathrm{Fe}, \mathrm{Zn}$, and crude protein content. Seven discriminant variates were obtained, of which the first five were characterized by Eigen values higher than 1 . The first canonical discriminant variate accounts for $49.0 \%$ of the total variation, and the other four for $46.7 \%$, which makes a total of $95.7 \%$ of total variability. From the means of canonical variables, it can be seen that the first canonical discriminant variate best discriminated the coppice populations Cazin and Petrova gora from the other high-forest populations, while the 
second variate best discriminated the Macelj and Tisovec population from the Bosiljevo, Pazin, and Combai populations (Figure 4). This pattern was primarily determined by water and $\mathrm{Mg}$ content, which most strongly influenced the first canonical discriminant variate. From Figures 3 and 4, it is evident that the studied populations can be better distinguished based on the chemical composition of kernels than on morphological traits of the nuts. This was also corroborated by the results of classification discriminant analysis. The overall classification rate for chemical analysis was $94.2 \%$. The highest percent of correctly classified individuals was observed in the Macelj, Tisovec, and Petrova gora populations $(100 \%)$, and the lowest in the Pazin population $(80 \%)$.

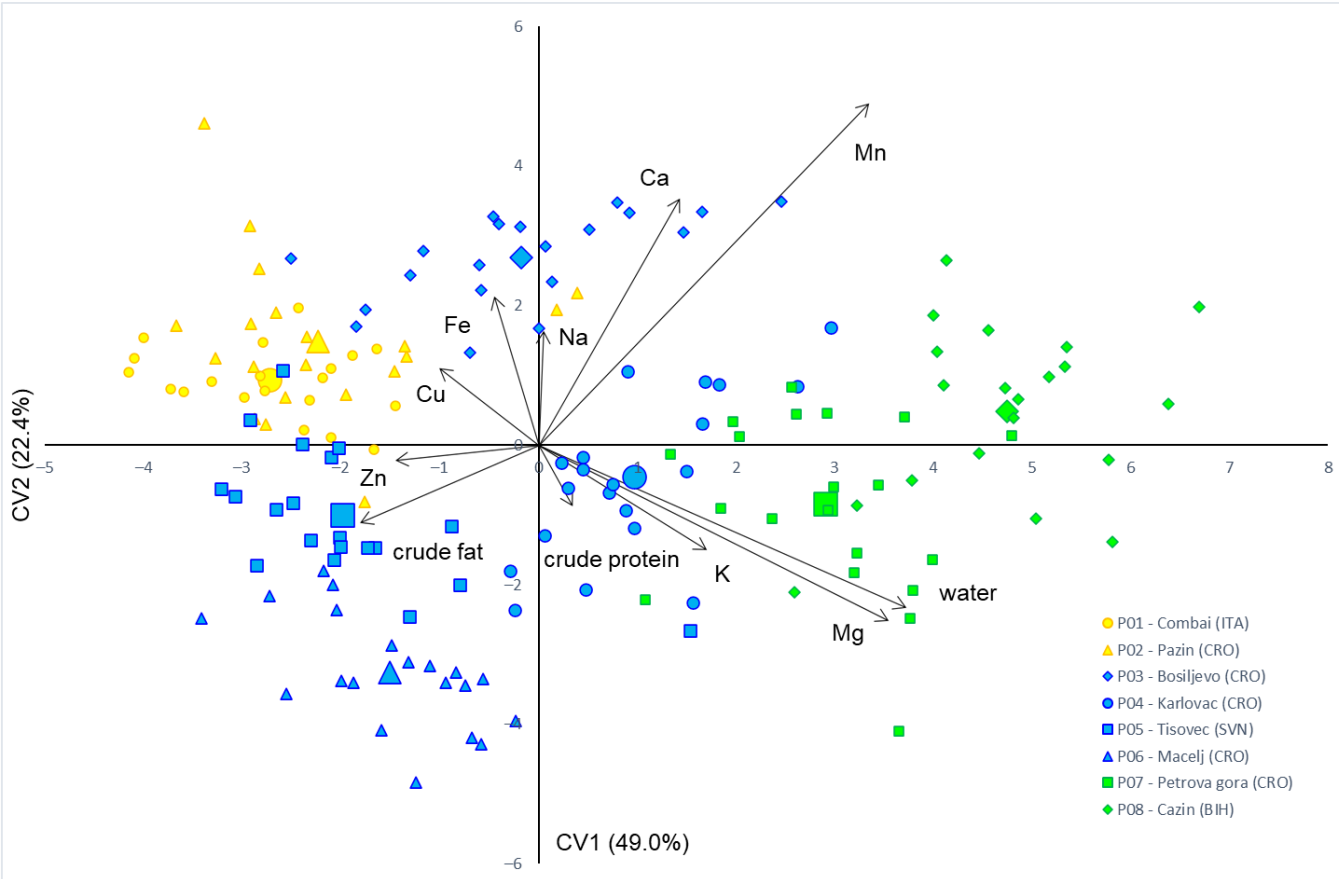

Figure 4. Discriminant analysis of eight sweet chestnut populations based on 11 chemical traits that were the most useful for maximum discrimination between studied populations. Each individual tree is indicated by a small sign, while the population barycenters are represented by larger ones. The color of the signs is related to the biogeographical region and management type: yellow color-subMediterranean high-forest populations; blue color-continental high-forest populations; and green color-continental coppice populations. Canonical discriminant variate $(\mathrm{CV})$.

In both cluster analyses, morphometric and chemical, the same pattern of population grouping was found (Figures S2 and S3). The studied populations were divided into two groups, of which the first included coppice populations of Petrova gora and Cazin, and the other high-forest populations Bosiljevo, Combai, Tisovec, Macelj, and Karlovac.

In order to determine whether the observed phenotypic and chemical variability was caused by geographical (IBD) or environmental distances (IBE) between the studied populations, the Mantel test was performed. Population-level pairwise morphological and chemical distance was not related to the geographic distance between populations (morphological $\mathrm{r}=-0.078, p=0.463$; chemical $\mathrm{r}=0.198, p=0.150$ ), nor to environmental distance (morphological $r=0.232, p=0.149$; chemical $r=0.170, p=0.210$ ). Finally, pairwise morphological and chemical distance matrices were strongly correlated $(r=0.529, p<0.01)$.

\section{Discussion}

\subsection{Nut Morphometric Traits}

Our results clearly demonstrate a significant morphological diversity of sweet chestnut nuts from high-forest stands and extensively managed coppices. The mean values of specific traits that describe the size and shape of the sweet chestnut fruits detected in this study 
were similar to those reported in flora and dendrology textbooks [68-71] or morphometric studies [46-51]. However, the marginal values for particular traits herein were much wider than in previously published research papers. Those differences probably resulted from the size of the sample collection and the size of the study area. Nevertheless, mean nut sizes of wild sweet chestnut populations in this and other studies were significantly lower compared to those of cultivated sweet chestnut varieties from orchards [24-32,44,45]. In addition, nuts from cultivated sweet chestnut trees from orchards were characterized by a significantly lower number of endocarp intrusions into the kernel. These findings are not surprising since chestnut trees in orchards are the result of the long artificial selection process, which eventually resulted in a large number of varieties with superior nut properties, such as size, taste, and ease of peeling.

The least variable traits analyzed in this research were those describing the shape of the nut and hilum. In addition, lower coefficients of variation were found for the height and width of the nut, whereas thickness of the nut showed moderate variation. The somewhat higher coefficient of variation for nut thickness compared to the values calculated for nut height and width can be explained by the number of nuts in chestnut burrs [51]. The cupules most often contain two or three nuts, but their number can vary from one to seven, which can end up significantly influencing their thickness. High values of the coefficient of variation were obtained for the trait of nut mass. The number and length of endocarp intrusions into the kernel, and their relation to nut thickness have been shown to be the most variable traits analyzed. Earlier morphometric studies of sweet chestnut populations reveal similar coefficients of variation for specific nut and kernel morphological traits [46-51].

All of the studied populations were highly variable, and there were no populations that stood out. However, these results are not in line with our earlier published data on microsatellite diversity of sweet chestnut populations in the same area [12]. In the mentioned study, the genetic diversity of peripheral chestnut populations in continental Croatia and Bosnia and Herzegovina was reduced. These results were attributed to successive founding events during the postglacial recolonization. The similar level of morphological variability in all populations studied herein can be explained by phenotypic plasticity that enables sweet chestnut trees to survive, grow, and reproduce under different environmental conditions [72-74]. Higher diversity of microhabitat conditions within those two peripheral populations of sweet chestnut finally resulted in a higher degree of morphological variability. In addition, the different age of the stumps in coppice populations may also have influenced the level of intrapopulation variability. The absence of association between genetic and phenotypic levels of variation when comparing genetic heterozygosity with coefficients of variation of the studied morphological traits of particular populations was reported by Douaihy et al. [75] for Juniperus excelsa M. Bieb.

High natural variability in nut dimensions and shapes was observed within and among populations of sweet chestnut. Similar to the present study, previous studies demonstrated that natural populations of sweet chestnut were characterized by high morphological $[48,50,51]$ and genetic $[9,11,12]$ variability, and that variability was higher within populations than among them. In general, variation in nut attributes (shape, length, width, thickness, and mass) of the sweet chestnut has been related to the genetic origin and environmental conditions [46-51]. Nevertheless, in our study, nut traits related to the dimensions were more connected to the population management type. Natural stands were characterized by larger nuts, and coppice populations by smaller nuts.

Statistical models that we use to assess variability among and within populations usually define "effect of error" as variability of leaves or fruits within the same tree. If the sampling was correctly conducted according to standardized protocols, this effect should not exceed the "effect of tree", at least when it pertains to leaves [76,77]. Nevertheless, our results suggest that, in the case of fruits, the "effect of tree" is responsible for a greater part of total variability than the "effect of error", which tells us that trees within populations are better differentiated in terms of dimensions and shape than individual fruits of the same tree. Similar results were also obtained by other authors who study morphological 
variability of the fruits of sweet chestnut $[48,50,51]$ and other tree species, such as the field elm [78] and service tree [79]. It follows that leaves compared to fruit within the same tree are under a much higher influence of microenvironmental factors. It has also been shown that fruits are better suitable for determining intra- and interpopulation variability in sweet chestnut compared to leaves [80].

\subsection{Kernel Chemical Composition}

As far as the proximate composition and macro- and micronutrients of sweet chestnut kernels from high-forest stands and managed coppices are concerned, the data available in the literature are still missing. On the other hand, there are many published research papers on the kernel chemical composition of traditional sweet chestnut varieties [32-45]. Overall, our results confirmed that chestnuts contain high levels of carbohydrates and low amounts of crude protein and, unlike typical nuts (walnuts, almonds, hazelnuts), low levels of crude fat. Due to a high starch content, in their chemical composition, chestnuts are more similar to cereals, hence the name "bread tree" for this species. The results of the chemical analysis herein were quite close to the data reported by other authors for cultivated varieties [32-45]. However, the distribution range in our study was much wider. Our previous results on a relatively small number of samples have confirmed the differences in total carbohydrate content between the kernels of cultivated and wild sweet chestnut trees [44]. The kernels of cultivated trees were characterized by higher values for total carbohydrates compared to the kernels of wild trees. This was confirmed in this study as well, as the values of total carbohydrates were lower than those for traditional chestnut varieties from the same area. Such insights are not surprising because the kernels of cultivated varieties are most often described as very sweet due to the high sugar content [81].

This research has found significant differences between the natural populations in the analyzed chemical composition of sweet chestnut kernels. In general, coppice populations have somewhat higher water content in comparison with high-forest populations. Furthermore, we did not find any differences in the chemical composition of kernels between continental and sub-Mediterranean populations. However, from the ecological point of view, those populations (P02 and P03) that were characterized by the carbonate substrate, covered with a deep soil layer, have somewhat higher amounts of $\mathrm{Ca}$ and micro-nutrients $\mathrm{Cu}$ and Fe. Similarly, several authors found that soil conditions can affect the chemical composition of the kernels of cultivated sweet chestnut varieties. For instance, Montaña Míguelez et al. [33] reported high protein content in chestnuts from the region characterized by schistose soils. Furthermore, Borges et al. [37] found that some regions in Portugal produced nuts with lower moisture content, which was attributed to the soil genesis of the studied area. These authors pointed out that different types of parent rock material and soil can affect the root zone aeration and the tree water potential, which can eventually also affect the chemical composition of the chestnuts. It was also demonstrated that the area of production has a higher influence on the physicochemical variables of chestnuts than the variety, particularly on the mineral composition [38]. It was also found that chemical heterogeneity within the same variety can be affected by management practices. Linhares et al. [82] and Mota et al. [83] reported that different irrigation systems in fruit orchards can affect chestnut quality concerning its chemical composition and nut size. These studies found that non-irrigated plantations resulted in smaller and sweeter chestnuts compared to irrigated plantations.

\subsection{Population Structure and Correlations between Morphological, Chemical, Geographical, and Environmental Distances}

A molecular study based on SSR markers showed that sweet chestnut from the Prealps in Italy, Slovenia, Croatia, and the northwestern part of Bosnia and Herzegovina can be classified into two different genetic groups [12]. In that study, coastal and island populations of sweet chestnut appeared as a separate group, being the most genetically differentiated. These findings were attributed to the proximity of sweet chestnut orchards and gene 
flow between natural and cultivated populations. However, the present study could not recover the two genetic groups mentioned above, and the collection studied here was divided into two clusters probably related to the management type-coppice and highforest. The first group of populations was associated with smaller nuts with higher water and $\mathrm{Mg}$ content, and the second group with larger nuts with lower water and $\mathrm{Mg}$ content. Differences between different types of production practices have also been described for Greek populations of sweet chestnut [47]. In contrast, leaf morphometric parameters were of limited use in studying variation and relationships of natural chestnut populations, when sampling is executed in situ [80], i.e., not in common garden experiments. It was concluded that leaf morphometry did not possess adequate discriminative capabilities to distinguish populations and population management types.

It is well known that different factors are involved in phenotypic divergences of plant populations-natural selection, gene flow, genetic drift, and, at a lower frequency, the acquisition of novel traits through mutations [84], and that phenotypic differences between plant populations may be correlated with geographical or environmental differences [85]. However, when we tested IBD and IBE patterns, we found no significant correlations. Our results revealed that management type can exceed genetic and environmental influences on the nut size and kernel nutritional value. However, we cannot completely exclude the possibility that the environment influences population variability in morphological and chemical nut traits. A characteristic of chestnut coppices compared to high-forests is short rotations and their intensive use. It has been reported that this cultivation method greatly depletes the soil and that such forests are extremely acidophilic $[14,15,17,18]$. In such forests, it is almost impossible to find trees from seed, and usually many stumps are old or dying out. Good quality, flat trees of larger dimensions are completely absent, and the predominant trees are low, stunted and bumpy. In addition to affecting the appearance of the trees, changes in the habitat, such as soil acidification, can significantly influence the size and chemical composition of the chestnuts. Besides, differences in morphology and chemical composition of the nuts can be due to the management type or physiological function during the growth forms. In coppice forests, the large root and the large amounts of nutrients stored in them lead to a culmination of height increment already during the first several years after the trees are felled. Although such plants start bearing nuts very quickly, already in the first year, they allocate the nutrients primarily to vegetative growth, which may ultimately influence the size of the nuts and their chemical composition.

\section{Conclusions}

Overall, our results confirmed significant population differentiation of sweet chestnut in the study area. All populations in this research had an equal level of morphological and chemical diversity. The research did not establish that morphological and chemical distances between populations correlate with geographical or environmental distances. However, the Mantel test identified significant correlations between the morphological and chemical distance matrices. Furthermore, multivariate statistical methods established that populations are grouped by management type. Nevertheless, additional research is required to establish how the management type affects fruit morphology and chemical composition of sweet chestnut kernels.

Supplementary Materials: The following are available online at https: / www.mdpi.com/article/ 10.3390 / f13010055/s1, Figure S1. Measured nut traits: nut height (NH); nut width (NW); distance from nut base to the point of maximum nut width (PMNW); nut thickness (NT); hilum length (HL); hilum width (HW); number of kernels (NK); number of intrusions of the seed coat into the kernel (NI); length of the longest intrusion of the seed coat into the kernel (LI). Figure S2. Tree diagram of the cluster analysis based on ten nut and kernel morphometric traits in studied sweet chestnut (Castanea sativa Mill.) populations. The unweighted pair-group method with arithmetic mean (UPGMA) was used to join the clusters, and the Euclidean distance to define the distance between the studied populations. Figure S3. Tree diagram of the cluster analysis based on 13 kernel chemical traits in studied sweet chestnut (Castanea sativa Mill.) populations. The unweighted pair-group method 
with arithmetic mean (UPGMA) was used to join the clusters, and the Euclidean distance to define the distance between the studied populations. Table S1. Pearson correlation coefficients between ten morphometric variables and scores of the first five principal components. Table S2. Pearson correlation coefficients between 13 chemical variables and scores of the first five principal components. Table S3. Results of the stepwise discriminant analyses for morphometric traits. Table S4. Results of the stepwise discriminant analyses for chemical traits.

Author Contributions: Conceptualization, I.P., N.V., Z.L., Z.Š. and M.I.; methodology, I.P., N.V., Z.L., Z.Š. and M.I.; software, I.P. and Z.Š.; investigation, I.P., N.V., Z.L., Z.Š. and M.I.; writing-original draft preparation, I.P.; writing—review and editing, N.V., Z.L., Z.Š. and M.I.; visualization, I.P. All authors have read and agreed to the published version of the manuscript.

Funding: This research was funded by Croatian Science Foundation (grant number IP-2018-01-1295) and University of Zagreb financial support.

Institutional Review Board Statement: Not applicable.

Informed Consent Statement: Not applicable.

Data Availability Statement: Not applicable.

Conflicts of Interest: The authors declare no conflict of interest. The funders had no role in the design of the study; in the collection, analyses, or interpretation of data; in the writing of the manuscript, or in the decision to publish the results.

\section{References}

1. Bottacci, A. Castanea sativa Miller 1768. In Enzyklopädie der Holzgewächse. Handbuch und Atlas der Dendrologie; Roloff, A., Schütt, P., Lang, U.M., Stimm, B., Eds.; Wiley-VCH: Weinheim, Germany, 1998; Volume 3, pp. 1-9.

2. Fernández-López, J.; Alía, R. Technical Guidelines for Genetic Conservation and Use for Chestnut (Castanea sativa Mill.); EUFORGEN International Plant Genetic Resources Institute: Rome, Italy, 2003; p. 6.

3. Conedera, M.; Krebs, P.; Tinner, W.; Prandella, M.; Torriani, D. The cultivation of Castanea sativa (Mill.) in Europe, from its origin to its diffusion on a continental scale. Veg. Hist. Archaeobot. 2004, 13, 161-179. [CrossRef]

4. Conedera, M.; Krebs, P. History, present situation and perspective of chestnut cultivation in Europe. Acta Hort. 2008, 784, 23-27. [CrossRef]

5. Krebs, P.; Conedera, M.; Pradella, M.; Torrioni, D.; Felber, M.; Tinner, W. Quaternary refugia of sweet chestnut (Castanea sativa Mill.): An extended palynological approach. Veg. Hist. Archaeobot. 2004, 13, 145-160. [CrossRef]

6. Villani, F.; Pigliucci, M.; Benedettelli, S.; Cherubini, M. Genetic differentiation among Turkish chestnut (Castanea sativa Mill.) populations. Heredity 1991, 66, 131-136. [CrossRef]

7. Villani, F.; Ansotta, A.S.; Cherubini, M.; Cesaroni, D.; Sbordoni, V. Genetic structure of natural populations of Castanea sativa in Turkey: Evidence of a hybrid zone. J. Evol. Biol. 1999, 12, 233-244. [CrossRef]

8. Martín, M.A.; Mattioni, C.; Molina, J.R.; Alvarez, J.B.; Cherubini, M.; Herrera, M.A.; Villani, F.; Martín, L.M. Landscape genetic structure of chestnut (Castanea sativa Mill.) in Spain. Tree Genet. Genomes 2012, 8, 127-136. [CrossRef]

9. Mattioni, C.; Martín, M.A.; Pollegioni, P.; Cherubini, M.; Villani, F. Microsatellite markers reveal a strong geographical structure in European populations of Castanea sativa (Fagaceae): Evidence for multiple glacial refugia. Am. J. Bot. 2013, 100, 951-961. [CrossRef]

10. Mattioni, C.; Martin, M.A.; Chiocchini, F.; Cherubini, M.; Gaudet, M.; Pollegioni, P.; Velichkov, I.; Jarman, R.; Chambers, F.M.; Paule, L.; et al. Landscape genetics structure of European sweet chestnut (Castanea sativa Mill): Indications for conservation priorities. Tree Genet. Genomes 2017, 13, 39. [CrossRef]

11. Lusini, I.; Velichkov, I.; Pollegioni, P.; Chiocchini, F.; Hinkov, G.; Zlatanov, T.; Cherubini, M.; Mattioni, C. Estimating the genetic diversity and spatial structure of Bulgarian Castanea sativa populations by SSRs: Implications for conservation. Conserv. Genet. 2014, 15, 283-293. [CrossRef]

12. Poljak, I.; Idžojtić, M.; Šatović, Z.; Ježić, M.; Ćurković-Perica, M.; Simovski, B.; Acevski, A.; Liber, Z. Genetic diversity of the sweet chestnut (Castanea sativa Mill.) in Central Europe and the western part of the Balkan Peninsula and evidence of marron genotype introgression into wild populations. Tree Genet. Genomes 2017, 13, 18. [CrossRef]

13. Anić, M. O Rasprostranjenosti Evropskog Pitomog Kestena s Osobitim Obzirom na Nezavisnu Državu Hrvatsku i Susjedne Zemlje; Tiskara C. Albrecht (P. Acinger): Zagreb, Croatia, 1942; p. 142.

14. Medak, J. Fitocenološke Značajke Šuma Pitomog Kestena u Sjeverozapadnoj Hrvatskoj. Master's Thesis, Faculty of Forestry and Wood Technology, University of Zagreb, Zagreb, Croatia, 5 December 2004.

15. Medak, J. Šumske Zajednice i Staništa Pitomog Kestena (Castanea sativa Mill.) u Hrvatskoj. Ph.D. Thesis, Faculty of Forestry and Wood Technology, University of Zagreb, Zagreb, Croatia, 18 December 2009. 
16. Conedera, M.; Tinner, W.; Krebs, P.; de Rigo, D.; Caudullo, G. Castanea sativa in Europe: Distribution, habitat, usage and threats. In European Atlas of Forest Tree Species; San-Miguel-Ayanz, J., de Rigo, D., Caudullo, G., Houston Durrant, T., Mauri, A., Eds.; Publ. Off. EU: Luxembourg, 2016; pp. 78-79.

17. Conedera, M.; Manetti, M.C.; Giudici, F.; Amorini, E. Distribution and economic potential of the sweet chestnut (Castanea sativa Mill.) in Europe. Ecol. Medit. 2004, 30, 179-193. [CrossRef]

18. Anić, M. Pitomi kesten u Zagrebačkoj gori. Glas. Šum. Pokuse 1940, 7, 103-312.

19. Lauteri, M.; Monteverdi, M.C.; Sansotta, A.; Cherubini, M.; Spaccino, L.; Villani, F.; Küçük, M. Adaptation to drought in European chestnut. Evidences from a hybrid zone and from controlled crosses between drought and wet adapted populations. Acta Hortic. 1998, 494, 345-353. [CrossRef]

20. Martín, M.A.; Mattioni, C.; Cherubini, M.; Taurchini, D.; Villani, F. Genetic diversity in European chestnut populations by means of genomic and genic microsatellite markers. Tree Genet. Genomes 2010, 6, 735-744. [CrossRef]

21. Míguez-Soto, B.; Fernández-Cruz, J.; Fernández-López, J. Mediterranean and Northern Iberian gene pools of wild Castanea sativa Mill. are two differentiated ecotypes originated under natural divergent selection. PLoS ONE 2019, 14, e211315. [CrossRef]

22. Mattioni, C.; Cherubini, M.; Micheli, E.; Villani, F.; Bucci, G. Role of domestication in shaping Castanea sativa genetic variation in Europe. Tree Genet. Genomes 2008, 4, 563-574. [CrossRef]

23. Aravanopoulos, F.A. Do silviculture and forest management affect the genetic diversity and structure of long-impacted forest tree populations? Forests 2018, 9, 355. [CrossRef]

24. Borghetti, M.; Menozzi, P.; Vendramin, G.G.; Giannini, R. Morphological variation in chestnut fruits (Castanea sativa Mill.) in Tuscany (Italy). Silvae Genet. 1986, 35, 124-128.

25. Pereira-Lorenzo, S.; Fernández-López, J.; Morengo-González, J. Variability and grouping of Northwestern Spanish chestnut cultivars. I. Morphological traits. J. Am. Soc. Hortic. 1996, 121, 183-189. [CrossRef]

26. Pereira-Lorenzo, S.; Díaz-Hernández, M.B.; Ramos-Cabrer, A. Use of highly discriminating morphological characters and isozymes in the study of Spanish chestnut cultivars. J. Am. Soc. Hortic. 2006, 131, 770-779. [CrossRef]

27. Pereira-Lorenzo, S.; Fernández-López, J. Description of 80 cultivars and 36 clonal selections of chestnut (Castanea sativa Mill.) from Northwestern Spain. Fruit Var. J. 1997, 51, 13-27.

28. Queijeiro, J.M.; De la Montaña, J.; Míguez, M. Identification and morphological description of cultivars of chesnut (Castanea sativa Mill.) of the region of Verín-Monterrei (Ourense, Spain). J. Am. Pomol. Soc. 2006, 60, 37-45. [CrossRef]

29. Ramos-Cabrer, A.M.; Pereira-Lorenzo, S. Genetic relationship between Castanea sativa Mill. trees from north-western to south Spain based on morphological traits and isoenzymes. Genet. Resour. Crop Evol. 2005, 52, 879-890. [CrossRef]

30. Álvarez-Álvarez, P.; Barrio-Anta, M.; Diéguez-Aranda, U. Differentiation of sweet chestnut (Castanea sativa Mill.) cultivars by leaf, nut and burr dimensions. Forestry 2006, 79, 149-158. [CrossRef]

31. Furones-Pérez, P.; Fernández-López, J. Morphological and phenological description of 38 sweet chestnut cultivars (Castanea sativa Miller) in a contemporary collection. Span. J. Agric. Res. 2009, 7, 829-843. [CrossRef]

32. Ertan, E. Variability in leaf and fruit morphology and in fruit composition of chestnuts (Castanea sativa Mill.) in the Nazilli region of Turkey. Genet. Resour. Crop Evol. 2007, 54, 691-699. [CrossRef]

33. De La Montaña Míguelez, J.; Miguez Bernàndez, M.; Garcia Quejeiro, J.M. Composition of varieties of chestnuts from Galicia (Spain). Food Chem. 2004, 84, 401-404. [CrossRef]

34. Botta, R.; Akkak, A.; Guaraldo, P.; Bounous, G. Genetic characterization and nut quality of chestnut cultivars from Piemonte (Italy). Acta Hortic. 2005, 693, 395-401. [CrossRef]

35. Pereira-Lorenzo, S.; Ramos-Cabrer, A.M.; Díaz-Hernández, M.B.; Ciordia-Ara, M.; Ríos-Mesa, D. Chemical composition of chestnut cultivars from Spain. Sci. Hortic. 2006, 107, 306-314. [CrossRef]

36. Borges, O.P.; Carvalho, J.S.; Correia, P.R.; Silva, A.P. Lipid and fatty acid profiles of Castanea sativa Mill. chestnuts of 17 native Portuguese cultivars. J. Food Compost. Anal. 2007, 20, 80-89. [CrossRef]

37. Borges, O.; Gonçalves, B.; Carvalho, J.L.S.; Correia, P.; Silva, A.P. Nutritional quality of chestnut (Castanea sativa Mill.) cultivars from Portugal. Food Chem. 2008, 106, 976-984. [CrossRef]

38. Peña-Méndez, E.M.; Hernández-Suárez, M.; Díaz-Romero, C.; Rodríguez-Rodríguez, E. Characterization of various chestnut cultivars by means of chemometrics approach. Food Chem. 2008, 107, 537-544. [CrossRef]

39. Barreira, J.C.M.; Casal, S.; Ferreira, I.C.F.R.; Oliveira, M.B.P.P.; Pereira, J.A. Nutritional, fatty acid and triacylglycerol profiles of Castanea sativa Mill. cultivars: A compositional and chemometric approach. J. Agric. Food Chem. 2009, 57, 2836-2842. [CrossRef]

40. Barreira, J.C.M.; Casal, S.; Ferreira, I.C.F.R.; Peres, A.M.; Pereira, J.A.; Oliviera, M.B.P.P. Chemical characterization of chestnut cultivars from three consecutive years: Chemometrics and contribution for authentication. Food Chem. Toxicol. 2012, 50, 2311-2317. [CrossRef]

41. Sacchetti, G.; Neri, L.; Dimitri, G.; Mastrocola, D. Chemical composition and functional properties of three sweet chestnut (Castanea sativa Mill.) ecotypes from Italy. Acta Hortic. 2009, 844, 41-46. [CrossRef]

42. Neri, L.; Dimitri, G.; Sacchetti, G. Chemical composition and antioxidant activity of cured chestnuts from three sweet chestnut (Castanea sativa Mill.) ecotypes from Italy. J. Food Compost. Anal. 2010, 23, 23-29. [CrossRef]

43. Mert, C.; Ertürk, Ü. Chemical compositions and sugar profiles of consumed chestnut cultivars in Marmara region, Turkey. Not. Bot. Horti. Agrobot. Cluj-Napoca 2017, 45, 203-207. [CrossRef] 
44. Poljak, I.; Vahčić, N.; Gačić, M.; Idžojtić, M. Morphology and chemical composition of fruits of the traditional Croatian chestnut variety 'Lovran Marron'. Food Technol. Biotechnol. 2016, 54, 189-199. [CrossRef]

45. Poljak, I.; Vahčić, N.; Vidaković, A.; Tumpa, K.; Žarković, I.; Idžojtić, M. Traditional sweet chestnut and hybrid varieties: Chemical composition, morphometric and qualitative nut characteristics. Agronomy 2021, 11, 516. [CrossRef]

46. Villani, F.; Pigliucci, M.; Lauteri, M.; Cherubini, M. Congruence between genetic, morphometric, and physiological data on differentiation of Turkish chestnut (Castanea sativa). Genome 1992, 35, 251-256. [CrossRef]

47. Aravanopoulos, F.A.; Drouzas, A.D.; Alizoti, P.G. Electrophoretic and quantitative variation in chestnut (Castanea sativa Mill.) in Hellenic populations in old-growth natural and coppice stands. For. Snow Landsc. Res. 2001, 76, 429-434.

48. Bolvanský, M.; Užík, M. Morphometric variation and differentiation of European chestnut (Castanea sativa) in Slovakia. Biologia 2005, 60, 423-429.

49. Solar, A.; Podjavoršek, A.; Štampar, F.S. Fenotypic and genotypic diversity of European chestnut (Castanea sativa Mill.) in Slovenia-Opportunity for genetic improvement. Genet. Resour. Crop Evol. 2005, 52, 391-394. [CrossRef]

50. Idžojtić, M.; Zebec, M.; Poljak, I.; Medak, J. Variation of sweet chestnut (Castanea sativa Mill.) populations in Croatia according to the morphology of fruits. Sauteria 2009, 18, 232-333.

51. Poljak, I.; Idžojtić, M.; Zebec, M.; Perković, N. The variability of European sweet chestnut (Castanea sativa Mill.) in the region of northwest Croatia according to morphology of fruits. Šumar. List 2012, 136, 479-489.

52. AOAC International. Nuts and nut products-Preparation of test sample procedure. In AOAC Official Method 935.52; AOAC International: Washington, DC, USA, 1995.

53. AOAC International. Nuts and nut products-Moisture in nuts and nut products. In AOAC Official Method 925.40; AOAC International: Washington, DC, USA, 1995.

54. AOAC International. Nuts and nut products-Ash of nuts and nut products. In AOAC Official Method 950.49; AOAC International: Washington, DC, USA, 1995.

55. AOAC International. Nuts and nut products-Fat (crude) in nuts and nut products. In AOAC Official Method 948.22; AOAC International: Washington, DC, USA, 2000.

56. AOAC International. Nuts and nut products-Protein (crude) in nuts and nut products. In AOAC Official Method 950.48; AOAC International: Washington, DC, USA, 1995.

57. Oliveira, I.; Sousa, A.; Morais, J.S.; Ferreira, I.C.; Bento, A.; Estevinho, L.; Pereira, J.A. Chemical composition, and antioxidant and antimicrobial activities of three hazelnut (Corylus avellana L.) cultivars. Food Chem. Toxicol. 2008, 46, 1801-1807. [CrossRef]

58. Pereira, J.A.; Oliveira, I.; Sousa, A.; Ferreira, I.C.F.R.; Bento, A.; Estevinho, L.M. Bioactive properties and chemical composition of six walnut (Juglans regia L.) cultivars. Food Chem. Toxicol. 2008, 46, 2103-2111. [CrossRef]

59. Sokal, R.R.; Rohlf, F.J. Biometry: The Principles and Practice of Statistics in Biological Research, 4th ed.; W.H. Freeman and Co.: New York, NY, USA, 2012; p. 937.

60. StatSoft, Inc. STATISTICA (Data Analysis Software System), Version 13; StatSoft, Inc.: Tulsa, OK, USA, 2018.

61. R Core Team. R: A Language and Environment for Statistics in Biological Research, 4th ed.; R Foundation for Statistical Computing: Vienna, Austria, 2020. Available online: http:/ / www.r-project.org/index.html (accessed on 22 October 2021).

62. Hijmans, R.J.; Cameron, S.E.; Parra, J.L.; Jones, P.G.; Jarvis, A. Very high resolution interpolated climate surfaces for global land areas. Int. J. Climatol. 2005, 25, 1965-1978. [CrossRef]

63. Fick, S.E.; Hijmans, R.J. WorldClim 2: New $1 \mathrm{~km}$ spatial resolution climate surfaces for global land areas. Int. J. Climatol. 2017, 37, 4302-4315. [CrossRef]

64. Mantel, N. The detection of disease clustering and a generalized regression approach. Cancer Res. 1967, 27, 209-220.

65. Smouse, P.E.; Long, J.C.; Sokal, R. Multiple regression and correlation extensions of the Mantel test of matrix correspondence. Syst. Zool. 1986, 35, 627-632. [CrossRef]

66. Manly, B.F.J. Randomization, Bootstrap and Monte Carlo Methods in Biology, 3rd ed.; Chapman \& Hall/CRC, Taylor \& Francis Group: Boca Raton, FL, USA, 2007; p. 480.

67. Rohlf, F.J. NTSYS-pc: Numerical Taxonomy and Multivariate Analysis System, Version 2.2; Applied Biostatistics Inc.: New York, NY, USA, 2009; p. 44.

68. Krüssmann, G. Handbuch der Laubgehölze; Paul Parkey in Berlin und Hamburg, Verlag für Landwirtschaft, Veterinärmedizin, Gartenbau und Forstwesen: Berlin, Germany, 1960.

69. Herman, J. Šumarska Dendrologija; Stanbiro: Zagreb, Croatia, 1971; p. 470.

70. Hegi, G. Illustrierte Flora von Mitteleuropa; Verlag Paul Parey: Berlin und Hamburg, Germany, 1981; Volume 3, p. 504.

71. Idžojtić, M. Dendrology: Cones, Flowers, Fruits and Seeds; Elsevier-Academic Press: London, UK; San Diego, CA, USA; Cambridge, MA, USA; London, UK, 2019; p. 800.

72. Garland, T., Jr.; Kelly, S.A. Phenotypic plasticity and experimental evolution. J. Exp. Biol. 2006, 209, 2344-2361. [CrossRef]

73. Albert, C.H.; Thuiller, W.; Yoccoz, N.G.; Soudant, A.; Boucher, F.; Saccone, P.; Lavorel, S. Intraspecific functional variability: Extent, structure and sources of variation. J. Ecol. 2010, 98, 604-613. [CrossRef]

74. Gratani, L. Plant phenotypic plasticity in response to environmental factors. Adv. Bot. 2014, 2014, 208747. [CrossRef]

75. Douaihy, B.; Sobierajska, K.; Jasińska, A.K.; Boratyńska, K.; Ok, T.; Romo, A.; Machon, N.; Didukh, Y.; Dagher-Kharrat, M.B.; Boratyński, A. Morphological versus molecular markers to describe variability in Juniperus excelsa subsp. excelsa (Cupressaceae). AOB Plants 2012, 2012, plr003. [CrossRef] 
76. Cornelissen, J.H.C.; Lavorel, S.; Garnier, E.; Diaz, S.; Buchmann, N.; Gurvich, D.E.; Pooter, H. Handbook of protocols for standardised and easy measurement of plant functional traits worldwide. A handbook of protocols for standardised and easy measurement of plant functional traits worldwide. Aust. J. Bot. 2003, 51, 335-380. [CrossRef]

77. Miljković, D.; Stefanović, M.; Orlović, S.; Stanković Neđić, M.; Kesić, L.; Stojnić, S. Wild cherry (Prunus avium (L.) L.) leaf shape and size variations in natural populations at different elevations. Alp. Bot. 2019, 129, 163-174. [CrossRef]

78. Zebec, M.; Drvodelić, D.; Moro, M. Geometric morphometric analysis of fruit shape variability in continental populations of Ulmus minor Mill. sensu latissimo from Croatia. In Book of Abstracts, Natural Resources Green Technology E Sustainable Development 2; Radojčić Redovniković, I., Radošević, K., Jakovljević, T., Stojaković, R., Gaurina Srček, V., Erdec Hendrih, D., Eds.; Faculty of Food Technology and Biotechnology: Zagreb, Croatia, 2016; p. 119.

79. Poljak, I.; Vahčić, N.; Liber, Z.; Tumpa, K.; Pintar, V.; Zegnal, I.; Vidaković, A.; Valković, B.; Kajba, D.; Idžojtić, M. Morphological and chemical diversity and antioxidant capacity of the service tree (Sorbus domestica L.) fruits from two eco-geographical regions. Plants 2021, 10, 1691. [CrossRef]

80. Aravanopoulos, F.A. Phenotypic variation and population relationships of chestnut (Castanea sativa) in Greece, revealed by multivariate analysis of leaf morphometrics. Acta Hortic. 2005, 693, 233-240. [CrossRef]

81. Bounous, G. Following chestnut footprints (Castanea spp.)—Cultivation and culture, folklore and history, traditions and uses. Scripta Hort. 2009, 9, 72-84.

82. Linhares, I.; Martins, A.; Borges, O.; Guedes, C.; Seixas Sousa, V. Effect of irrigation and soil management practices on fruit production and quality in chestnut orchards of northern Portugal. Acta Hortic. 2005, 693, 701-706. [CrossRef]

83. Mota, M.; Pinto, T.; Vilela, A.; Marques, T.; Borges, A.; Caço, J.; Ferreira-Cardoso, J.; Raimundo, F.; Gomes-Laranjo, J. Irrigation positively affects the chestnut's quality: The chemical composition, fruit size and sensory attributes. Sci. Hortic. 2018, 238, 177-186. [CrossRef]

84. Wright, S. Evolution in Mendelian populations. Genetics 1931, 16, 97-159. [CrossRef] [PubMed]

85. DeWoody, J.; Trewin, H.; Taylor, G. Genetic and morphological differentiation in Populus nigra L.: Isolation by colonization or isolation by adaptation? Mol. Ecol. 2015, 24, 2641-2655. [CrossRef] [PubMed] 